\title{
Role of point defects in spinel Mg chalcogenide conductors
}

\author{
Pieremanuele Canepa, ${ }^{*, \dagger, \ddagger, \|}$ Gopalakrishnan Sai Gautam, $9, \dagger, \ddagger$ Danny Broberg,, \\ Shou-Hang Bo, ${ }^{\dagger}$ and Gerbrand Ceder ${ }^{*}, \dagger, \uparrow, \S$ \\ $\dagger$ †aterials Science Division, Lawrence Berkeley National Laboratory, Berkeley, CA 94720, \\ USA \\ $\ddagger$ Contributed equally to this work \\ I Department of Materials Science and Engineering, Massachusetts Institute of \\ Technology, Cambridge, MA 02139, USA \\ $\S$ Department of Materials Science and Engineering, University of California Berkeley, \\ Berkeley, CA 94720, USA \\ ||Current address: Department of Chemistry, University of Bath, Bath, BA2 7AY, UK. \\ E-mail: pcanepa@lbl.gov; gceder@berkeley.edu
}

\begin{abstract}
Close-packed chalcogenide spinels, such as $\mathrm{MgSc}_{2} \mathrm{Se}_{4}, \mathrm{MgIn}_{2} \mathrm{~S}_{4}$ and $\mathrm{MgSc}_{2} \mathrm{~S}_{4}$, show potential as solid electrolytes in Mg batteries, but are affected by non-negligible electronic conductivity, which contributes to self-discharge when used in an electrochemical storage device. Using first-principles calculations, we evaluate the energy of point defects as function of synthesis conditions and Fermi level to identify the origins of the undesired electronic conductivity. Our results suggest that Mg-vacancies and Mg-metal anti-sites (where Mg is exchanged with Sc or In) are the dominant point defects that can occur in the systems under consideration. While we find anion-excess conditions and
\end{abstract}


slow cooling to likely create conditions for low electronic conductivity, the spinels are likely to exhibit significant $n$-type conductivity under anion-poor environments, which are often present during high temperature synthesis. Finally, we explore extrinsic aliovalent doping to potentially mitigate the electronic conductivity in these chalcogenide spinels. The computational strategy is general and can be easily extended to other solid electrolytes (and electrodes) to aid in the optimization of the electronic properties of the corresponding frameworks.

\section{Introduction}

Chalcogenide materials, based on sulfur, selenium and tellurium, are used in a range of technological applications, including thermoelectric materials, ${ }^{1,2}$ semiconductors for light adsorbents and electronics, ${ }^{3-11}$ superconductors ${ }^{12-16}$ Li-ion battery materials, ${ }^{17-22}$ quantumdots, ${ }^{23-27}$ and more recently, topological insulators. ${ }^{28,29}$ Specifically, sulfides have already seen applications as solid electrolytes (or super-ionic conductors) in solid-state Li-ion batteries. ${ }^{19-21}$ The chalcogenide defect chemistry, either in terms of intrinsic point defects or extrinsic substitutional impurities, has often been deemed responsible for their respective figures of merit. ${ }^{30,31}$

Recently, ternary Mg-chalcogenide spinels were also identified as possible high mobility Mg-conductors. ${ }^{22}$ This is relevant for the possible development of Mg transport coatings or solid state electrolytes for Mg batteries, ${ }^{22}$ which have the potential to outperform Li-ion batteries in terms of energy density. ${ }^{32}$ The good $\mathrm{Mg}$ conductivity in the $\mathrm{MgSc}_{2} \mathrm{Se}_{4}, \mathrm{MgIn}_{2} \mathrm{~S}_{4}$ and

$\mathrm{MgSc}_{2} \mathrm{~S}_{4}$ spinels is, however, plagued by non-negligible electronic conductivity. ${ }^{22}$ Though the significant $\mathrm{Mg}$ ionic conductivity $\sigma_{\text {ionic }} \sim 0.1 \mathrm{mS} \mathrm{cm}{ }^{-1}$ (at $298 \mathrm{~K}$ ) observed in $\mathrm{MgSc}_{2} \mathrm{Se}_{4}$ (via ${ }^{25} \mathrm{Mg}$ magic angle spin solid-state NMR and AC impedance spectroscopy) the electronic conductivity of $\mathrm{MgSc}_{2} \mathrm{Se}_{4}$ is $\sim 0.04 \%$ of the ionic conductivity, ${ }^{22}$ and substantially larger than in other state-of-the-art alkali $(\mathrm{Li}$ and $\mathrm{Na})$ ion conductors $\left(\sigma_{\text {electronic }} / \sigma_{\text {ionic }} \quad 10^{-4}-10^{-6} \%\right) .{ }^{19}$ Analogous to studies in semi-conductor applications, ${ }^{33}$ both intrinsic and extrinsic structural 
defects can cause large variations in electron (hole) conductivity in ionic conductors. Thus, we explore the defect chemistry of $\mathrm{MgSc}_{2} \mathrm{Se}_{4}, \mathrm{MgSc}_{2} \mathrm{~S}_{4}$ and $\mathrm{MgIn}_{2} \mathrm{~S}_{4}$ using first-principles calculations and aim to understand how structural defects modulate the electronic properties in the bulk spinels, identify the origin of the undesired electronic conductivity, and propose practical remedies.

In detail, our calculations suggest that intrinsic point defects, such as $\mathrm{Sc}^{3+}$ substituting

on $\mathrm{Mg}^{2+}$ sites in $\mathrm{MgSc}_{2} \mathrm{~S}_{4}$ or $\mathrm{MgSc}_{2} \mathrm{Se}_{4}\left(\mathrm{Sc}_{\mathrm{Mg}}^{\bullet}\right.$ using the Kröger-Vink notation), and $\mathrm{In}_{\mathrm{Mg}}^{\bullet}$ and $\mathrm{Mg}_{\mathrm{In}}^{\prime}$ in $\mathrm{MgIn}_{2} \mathrm{~S}_{4}$, can give rise to significant electronic conductivity in these materials. Additionally, our data demonstrates that anion-rich and anion-poor synthesis conditions should give rise to qualitatively different defects, affecting the electronic (hole) conductivity of these materials. Finally, we demonstrate that understanding and controlling the defect chemistry of solid electrolytes (and cathode materials) is crucial in all aspects, such as tuning the respective synthesis conditions and optimizing the electronic and ionic conductivities.

\section{Methodology}

\subsection{Basics of defect chemistry}

The occurrence of a defect $X$ of charge $q$ in a solid relates to its formation energy $E^{f}\left[X^{q}\right]$ :

$$
E^{f}\left[X^{q}\right]=E_{t o t}\left[X^{q}\right]-E_{t o t}[b u l k]-\sum_{i} n_{i} \mu_{i}+q E_{F e r m i}+E_{c o r r}
$$

where, $E_{t o t}\left[X^{q}\right]$ and $E_{t o t}[b u l k]$ are the total energies of a supercell containing the defect $X$ and an un-defected supercell, respectively. ${ }^{33-37} n_{i}$ is the concentration of species $i$ added $\left(n_{i}>0\right)$ or removed $\left(n_{i}<0\right)$ to create defect $X . \mu_{i}$ is the chemical potential of species $i$, as determined by the set of phases in thermodynamic equilibrium with the solid of interest at $0 \mathrm{~K} . E_{F e r m i}$ is the Fermi energy of electrons in the structure, and $E_{\text {corr }}$ is the electrostatic correction term to account for spurious interactions among defects (i.e., with periodic images 
and the homogeneous background charge). Using Eq. 1, the defect formation energies $E^{f}\left[X^{q}\right]$ can be plotted as a function of the Fermi energy, $E_{\mathrm{Fermi}}$, as demonstrated in Figure 3.

In this work, we compute $E_{\text {corr }}$ using the Freysoldt correction scheme, ${ }^{33,38-40}$ which separates the electrostatic interactions into a short range (decaying to zero in a large supercell) and a long range ( $\sim \frac{1}{\varepsilon r}$ beyond the supercell boundaries) component. The dielectric constants $(\varepsilon)$ of the spinels utilized to approximate the long range part of the electrostatic interactions, are reported in Table S2. For a given defect, the value of $E_{\text {corr }}$ within the Freysoldt scheme $e^{38,39}$ is determined by the convergence of the short range potential to a constant value with increasing supercell size (as seen in Figure S1). Recently, Komsa et al. ${ }^{41}$ determined the Freysoldt scheme to be more efficient than other schemes, ${ }^{41-46}$ in terms of the supercell size required to achieve convergence, and quantified the average error of the Freysoldt correction to be $\sim 0.09 \mathrm{eV}$ in a variety of systems. ${ }^{41}$ Specifically for the $\mathrm{MgA}_{2} \mathrm{Z}_{4}$ spinels $(\mathrm{A}=\mathrm{Sc} / \mathrm{In}, \mathrm{Z}=\mathrm{S} / \mathrm{Se})$, we use a $2 \times 2 \times 2$ supercell of the conventional cubic cell, which contains 256 anions.

Throughout the article the Kröger-Vink notation is employed to identify the type of point defects in the $\mathrm{MgA}_{2} \mathrm{Z}_{4}$ spinels, including chalcogenide vacancies (e.g., Vacse), metal vacancies $\left(\mathrm{Vac}_{\mathrm{Mg}}, \mathrm{Vac}_{\mathrm{Sc}}\right)$, chalcogenide anti-sites $\left(\mathrm{Se}_{\mathrm{Mg}}, \mathrm{Se}_{\mathrm{Sc}}\right)$ and metal anti-sites $\left(\mathrm{Mg}_{\mathrm{Sc}}\right.$,

$\mathrm{Sc}_{\mathrm{Mg}}$ ). For example, $\mathrm{Sc}_{\mathrm{Mg}}^{\bullet}$ identifies a positively charged anti-site defect, where a $\mathrm{Sc}^{3+}$ atom replaces $\mathrm{Mg}^{2+}$. Similarly, $\mathrm{Vac}_{\mathrm{Mg}}^{\prime \prime}$ and $\mathrm{Mg}_{\mathrm{Sc}}^{\times}$represent a double-negatively charged vacancy on a Mg site and a neutral Mg anti-site on Sc, respectively.

\subsection{Charge neutrality}

Point defects can be neutral or charged specie. An example is shown in Figure S2, where the donor defect is positively charged for $(q=1) E_{\mathrm{Fermi}}<\epsilon(+/ 0)$, while for $E_{\mathrm{Fermi}}>\epsilon(+/ 0)$ the donor defect is neutral $(q=0)$. Thus, $\epsilon(+/ 0)$ is the thermodynamic defect transition level where two different charge states of a defect have the same $E_{f}$. The availability of electrons is set by the equilibrium Fermi level $E_{\text {Fermi }}^{e q}$, and the defect transition level sets the 


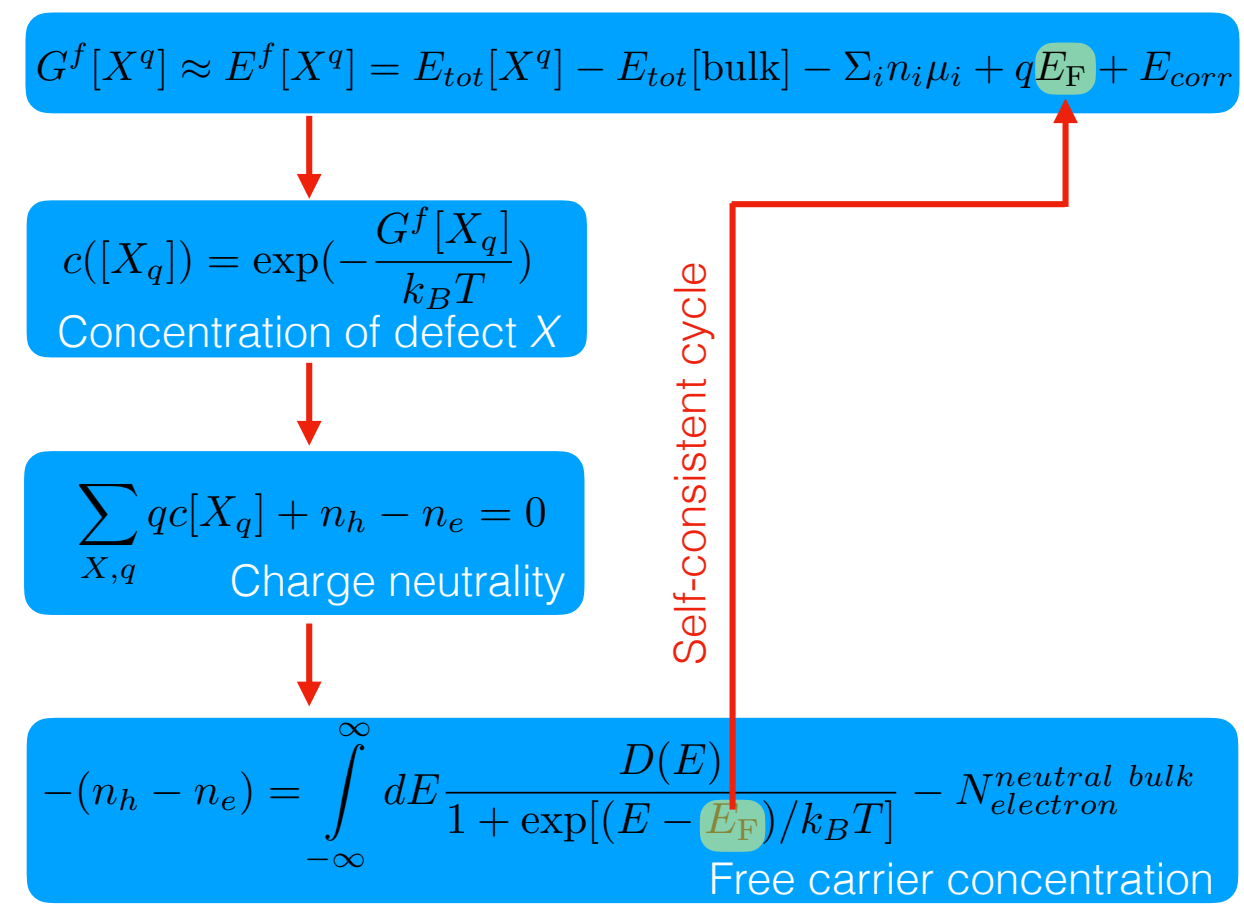

Figure 1: Self-consistent search of the equilibrium Fermi level $\left(E_{F}\right)$, defect $\left(c\left[X^{q}\right]\right)$ and free carrier $\left(n_{h}\right.$ and $\left.n_{e}\right)$ concentrations at a given temperature $(\mathrm{T})$. The chemical potentials $\left(\mu_{i}\right)$ are required as input parameters.

$E_{F e r m i}^{e q}$ within the band gap and in turn, the electronic conductivity of the structure.

When multiple defects and charge states are present in a structure, estimation of $E_{F e r m i}^{e q}$ requires a self-consistent search (as explained in Figure 1) by enforcing charge neutrality of the material, corresponding to $\sum_{X, q} q c\left[X^{q}\right]+n_{h}-n_{e}=0 . n_{h}\left(n_{e}\right)$ is the hole (electron) concentration, obtained by integrating the density of states (DOS, $D(E)$ in Figure 1) at a given Fermi level $\left(E_{F}\right)$ in the structure. $N_{\text {electron }}^{\text {neutral bul }}$ in Figure 1 is the total number of electrons in the neutral bulk cell. $c\left[X^{q}\right]$ is the concentration of defect $X^{q}$, stemming from the Gibbs energy of defect formation, $G_{f}\left[X^{q}\right] \approx E_{f}\left[X^{q}\right]$, as $c\left[X^{q}\right] \approx \exp \left(-E_{f}\left[X^{q}\right] / k_{B} T\right)$. The resulting $E_{F e r m i}^{e q}$ and defect concentrations correspond to thermodynamic equilibrium as a function of temperature. Note that in all the materials considered in this work (Section 4), we list a few defects as "dominant" owing to their low formation energies at $E_{F e r m i}^{e q}$.

Materials that are normally synthesized at a high temperature $\left(\sim 1273 \mathrm{~K}\right.$, for example $\left.{ }^{22}\right)$ and rapidly cooled to room temperature, may have their high temperature intrinsic defect 
concentrations "frozen-in" (or quenched) at room temperature, while the free carrier concentration $\left(n_{h}-n_{e}\right)$ changes with temperature, given the fixed defect concentration. A change in intrinsic defect concentration will require significant atomic diffusion, which is likely to be kinetically limited at low temperatures. Hence, for calculating defect concentrations and the Fermi level, we have considered two scenarios within the constraint of charge neutrality, $(i)$ defect concentrations, equilibrium Fermi level $\left(E_{F e r m i}^{e q}\right)$ and free electron/hole concentrations $\left(c[e / h]^{e q}\right)$ are self-consistently calculated at $300 \mathrm{~K}$ corresponding to equilibrium conditions (as in Figure 1), (ii) defect concentrations are quenched from a higher synthesis temperature while the resulting Fermi level $\left(E_{\text {Fermi }}^{\text {frozen }}\right)$ and free carrier concentrations $\left(c[e / h]^{\text {frozen }}\right)$ are computed at $300 \mathrm{~K}$. When quenched or frozen conditions are assumed, the defect concentrations are calculated self-consistently at a higher quench temperature (i.e. $1273 \mathrm{~K}$ with the procedure in Figure 1), and are not allowed to change when the Fermi level and the free-carrier concentrations are re-calculated at $300 \mathrm{~K}$. Since defect concentrations increase with increasing temperatures, the frozen approximation can quantify the possible deviations away from equilibrium in both defect and free carrier concentrations at $300 \mathrm{~K}$.

\subsection{Computational Details}

The total energies in Eq. 1 are obtained with Density Functional Theory ${ }^{47,48}$ (DFT) using the Perdew Burke Ernzerhof (PBE) functional within the spin-polarized Generalized Gradient Approximation, ${ }^{49}$ as implemented in the VASP code. ${ }^{50,51}$ Projector Augmented Wave theory ${ }^{52,53}$ and a plane-wave basis set with a cutoff of $520 \mathrm{eV}$ are used to describe the crystalline wave-functions, which are subsequently sampled on a dense (minimum of 1000 $k$-points per atom in reciprocal space) $\Gamma$-centered $k$-point mesh. ${ }^{54}$ The Python Materials Genomics (pymatgen) ${ }^{55}$ and the Python Charged Defect Toolkit (PyCDT) ${ }^{40}$ libraries are leveraged for input preparation and data analysis. For calculating the chemical potentials of the various species involved in the defect calculations, we utilize the Materials Project database $^{56}$ in addition to our own calculations. 
In order to sample the large chemical space of defects, we use the computationally inexpensive semi-local PBE exchange-correlation functional, especially because the nature of the valence and conduction bands of the spinels considered, which are populated by the chalcogen $(\mathrm{S} / \mathrm{Se})$ and the metal (In/Sc) states respectively, do not change with a higher level of theory, such as HSE06 ${ }^{57,58}$ (see Figure S3 ${ }^{59}$ in SI). To confirm this hypothesis we performed calculations of the low-lying defects in $\mathrm{MgIn}_{2} \mathrm{~S}_{4}$ (see Section 4.2) utilizing both GGA and HSE06, and the comparison is detailed in Section S9 of the SI. In general, PBE is known to underestimate the band gap in most solids (by at least $30 \%{ }^{60}$ ) when compared to HSE06 (Figure S3). For example, the PBE-computed direct band gaps are $\sim 1.77 \mathrm{eV}$, $\sim 1.56 \mathrm{eV}$, and $\sim 1.09 \mathrm{eV}$ for the $\mathrm{MgIn}_{2} \mathrm{~S}_{4}, \mathrm{MgSc}_{2} \mathrm{~S}_{4}$ and $\mathrm{MgSc}_{2} \mathrm{Se}_{4}$, respectively, while the magnitude of the gaps increase with HSE06, ${ }^{61} \sim 2.82 \mathrm{eV}$ in $\mathrm{MgIn}_{2} \mathrm{~S}_{4}, \sim 2.63 \mathrm{eV}$ in $\mathrm{MgSc}_{2} \mathrm{~S}_{4}$, and $\sim 2.03 \mathrm{eV}$ in $\mathrm{MgSc}_{2} \mathrm{Se}_{4}$ (Figure S3). Note that the band gaps decrease while moving down the chalcogenide group (i.e., $\mathrm{S} \rightarrow \mathrm{Se}$ ), under both PBE and HSE06 calculations.

\section{$3 \quad \mathrm{MgA}_{2} \mathrm{Z}_{4}$ structure and phase diagram}

The spinel structure $\mathrm{MgA}_{2} \mathrm{Z}_{4}$ (with $\mathrm{A}=\mathrm{In}$ or $\mathrm{Sc}$ and $\mathrm{Z}=\mathrm{S}$ or $\mathrm{Se}$ ), crystallizes with the anions in the face centered cubic (FCC) packing (space group: $F d \overline{3} m$ ). In "normal" spinel structures, the higher valent cations $\left(\mathrm{A}=\mathrm{In}^{3+}\right.$ or $\left.\mathrm{Sc}^{3+}\right)$, occupy octahedral (oct) sites $16 d$, as shown by the purple polyhedra in Figure 2c, and the $\mathrm{Mg}^{2+}$ occupy the tetrahedral (tet) $8 a$ sites (orange polyhedra). Few spinels, such as $\operatorname{MgIn}_{2} \mathrm{~S}_{4}$, can also exhibit "inversion", as experimentally observed by Gastaldi et al. ${ }^{62}$ where a fraction of $\mathrm{Mg}^{2+}$ ions in the $8 a$ exchange sites with the $\mathrm{In}^{3+}$ in $16 d$.

The ternary 0 K phase-diagrams of Figures 2a and b depict four phases: Se, MgSe, ScSe and $\mathrm{Sc}_{2} \mathrm{Se}_{3}$ that can be in thermodynamic equilibrium with the ternary $\mathrm{MgSc}_{2} \mathrm{Se}_{4}$ spinel, at different atomic chemical potentials $\left(\mu_{\mathrm{Se}}\right.$ and $\left.\mu_{\mathrm{Mg}}\right)$. Equivalent phase diagrams have been constructed for the Mg-In-S and Mg-Sc-S systems and are presented in Figure S4a and S4b 


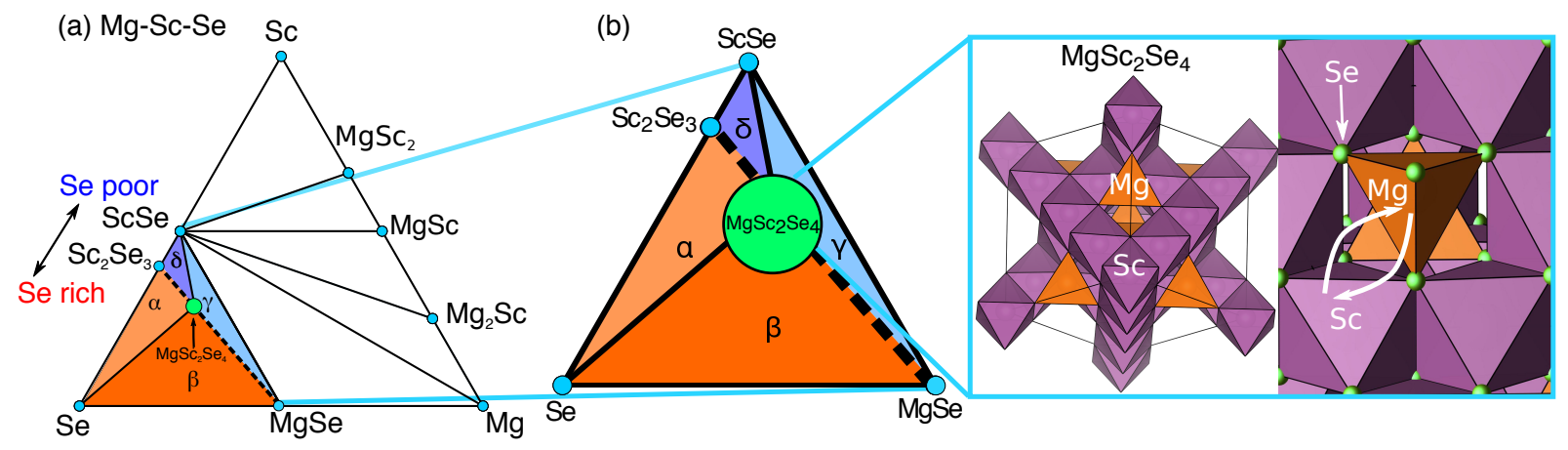

Figure 2: (a) Ternary Mg-Sc-Se phase-diagram at $0 \mathrm{~K}$ computed from DFT data combined with Materials Project, ${ }^{56}$ with (b) displaying a zoom-in of the concentration range of interest. (c) Crystal structure of a normal spinel, such as $\mathrm{MgSc}_{2} \mathrm{Se}_{4}$ identified in the phase diagrams of panels (a) and (b). The right fragment in (c) shows the scenario of spinel inversion (white arrows $\mathrm{Mg} \leftrightarrow \mathrm{Sc}$ ) in $\mathrm{MgSc}_{2} \mathrm{Se}_{4}$, leading to anti-site $\mathrm{MgSc}_{\mathrm{Sc}}$ and $\mathrm{Sc}_{\mathrm{Mg}}$ defects. Similar ternary phase diagrams for $\mathrm{MgIn}_{2} \mathrm{~S}_{4}$ and $\mathrm{MgSc}_{2} \mathrm{~S}_{4}$ are shown in Figure S4.

of the SI. The four different facets of Figure 2a and b, namely $\alpha \mathrm{MgSc}_{2} \mathrm{Se}_{4}-\mathrm{Se}_{-} \mathrm{Sc}_{2} \mathrm{Se}_{3}$ (light orange), $\beta \mathrm{MgSc}_{2} \mathrm{Se}_{4}$-Se-MgSe (dark orange), $\gamma \mathrm{MgSc}_{2} \mathrm{Se}_{4}$-MgSe-ScSe (dark violet), and $\delta$ $\mathrm{MgSc}_{2} \mathrm{Se}_{4}-\mathrm{ScSe}_{-} \mathrm{Sc}_{2} \mathrm{Se}_{3}$ (light violet), define the possible limiting chemical potential values ( $\mu_{i}$ of Eq. 1) for intrinsic point defect formation, such as vacancies (e.g., $\mathrm{Vac}_{\mathrm{Mg}}$ ) and anti-sites (e.g., $\mathrm{Mg}_{\mathrm{Sc}}$ ). Subsequently, the $\alpha$ and $\beta$ facets can be classified as "Se rich" domains, owing to elemental Se forming one of the bounding vertices of the respective facets, while $\gamma$ and $\delta$ are "Se poor". The dashed line in Figure $2 \mathrm{a}$ and b highlights the binary precursors, MgSe and $\mathrm{Sc}_{2} \mathrm{Se}_{3}$, which are used for the high-temperature synthesis $\left(\sim 1200{ }^{\circ} \mathrm{C}\right)$ of $\mathrm{MgSc}_{2} \mathrm{Se}_{4} \cdot{ }^{12}$ Off-stoichiometry of $\mathrm{MgSc}_{2} \mathrm{Se}_{4}$, will place the thermodynamic equilibrium during synthesis into one of the four facets ( $\alpha$ to $\delta$ ), which in turn can influence the $E_{f}\left[X^{q}\right]$ and the defect concentrations.

\section{Native defects}

\section{1 $\quad \mathrm{MgSc}_{2} \mathrm{Se}_{4}$}

Figure 3 shows the formation energies $E_{f}\left[X^{q}\right]$ of intrinsic defects in $\mathrm{MgSc}_{2} \mathrm{Se}_{4}$ obtained for the chemical potential in each of the four facets in the $\mathrm{Mg}$-Sc-Se system, namely $\mathrm{MgSc}_{2} \mathrm{Se}_{4}$-Se- 
$\mathrm{Sc}_{2} \mathrm{Se}_{3} \alpha$ (Figure 3a), MgSc $\mathrm{Se}_{4}$-Se-MgSe $\beta$ (Figure 3b), MgSc $\mathrm{Se}_{4}$-MgSe-ScSe $\gamma$ (Figure 3c), and $\mathrm{MgSc}_{2} \mathrm{Se}_{4}-\mathrm{MgSe}-\mathrm{Sc}_{2} \mathrm{Se}_{3} \delta$ (Figure 3d). The $y$-axis of each panel in Figure 3 plots the defect energy against the $E_{\mathrm{Fermi}}$ ( $x$-axis) in $\mathrm{MgSc}_{2} \mathrm{Se}_{4}$. The absolute value of the Fermi energy is referenced to the Valence Band Maximum (VBM) energy of the pristine $\mathrm{MgSc}_{2} \mathrm{Se}_{4}$ bulk. The zero of the $x$-axis is the VBM, with grey shaded regions being the valence $\left(E_{F e r m i}<0\right)$ and the conduction bands $\left(E_{F e r m i}>E_{g a p} \sim 1.09 \mathrm{eV}\right)$, respectively. The band gap spans the white area in all panels of Figure 3. In general, the defect levels with low formation energies in the band gap can considerably alter the intrinsic electronic conductivity of semiconductors and insulators, thus forming the region of interest in this analysis.

Facets $\alpha$ and $\beta$ (Figures 3a and b) are Se-rich, and show qualitatively similar defect energetics. For example, the defects with the lowest $E_{f}\left[X^{q}\right]$ are the $\mathrm{Sc}_{\mathrm{Mg}}, \mathrm{Mg}_{\mathrm{Sc}}$ and $\mathrm{Vac}_{\mathrm{Mg}}$ in both $\alpha$ and $\beta$. $\mathrm{Sc}_{2} \mathrm{Se}_{3}$ has been previously been detected as a prominent impurity in the synthesis of $\mathrm{MgSc}_{2} \mathrm{Se}_{4},{ }^{22}$ thus motivating the choice of facet $\alpha\left(\mathrm{MgSc}_{2} \mathrm{Se}_{4}-\mathrm{Se}_{-} \mathrm{Sc}_{2} \mathrm{Se}_{3}\right)$ to characterize the Se-rich domain. Similar conclusions are deduced by comparing the $\gamma$ and $\delta$ facets (Figure $3 \mathrm{c}$ and d), with comparable $E_{f}\left[X^{q}\right]$ for the low lying defects (e.g., $\mathrm{Sc}_{\mathrm{Mg}}$ ), and only the $\gamma$ phase is considered further to analyze the Se-poor domain. Analogous behaviors are also observed for $\mathrm{MgSc}_{2} \mathrm{~S}_{4}$ and $\mathrm{MgIn}_{2} \mathrm{~S}_{4}$, showing similar trends for the S-rich and S-poor domains (Figure S5 and S6 in the SI).

\section{Se-rich domain, $\alpha \mathrm{MgSc}_{2} \mathrm{Se}_{4}-\mathrm{Se}_{-} \mathrm{Sc}_{2} \mathrm{Se}_{3}$}

The dominant defects within the band gap of the Se-rich region are the charged $\mathrm{Sc}_{\mathrm{Mg}}^{\bullet}, \mathrm{Mg}_{\mathrm{Sc}}^{\prime}$ and $\mathrm{Vac}_{\mathrm{Mg}}^{\prime \prime}$ (dark blue, light green and dashed green lines, respectively, in Figure 3a). A charged defect always exchanges its excess (deficient) charge with the electron reservoir of the structure, whose energy is given by the Fermi energy. Thus, the $n$-type $\mathrm{Sc}_{\mathrm{Mg}}^{\bullet}$ exchanges the excess valence electron from $\mathrm{Sc}$ with the Fermi level of $\mathrm{MgSc}_{2} \mathrm{Se}_{4}$. Analogous considerations extend to the $p$-type $\mathrm{Mg}_{\mathrm{Sc}}^{\prime}$, where one electron is added to the anti-site from the Fermi level.

Given that the opposite charges of $\mathrm{Sc}_{\mathrm{Mg}}^{\bullet}, \mathrm{Mg}_{\mathrm{Sc}}^{\prime}$ and $\mathrm{Vac}_{\mathrm{Mg}}^{\prime \prime}$ can potentially charge-compensate 
(a) $\alpha \mathrm{MgSc}_{2} \mathrm{Se}_{4}-\mathrm{Se}_{-} \mathrm{Sc}_{2} \mathrm{Se}_{3}$

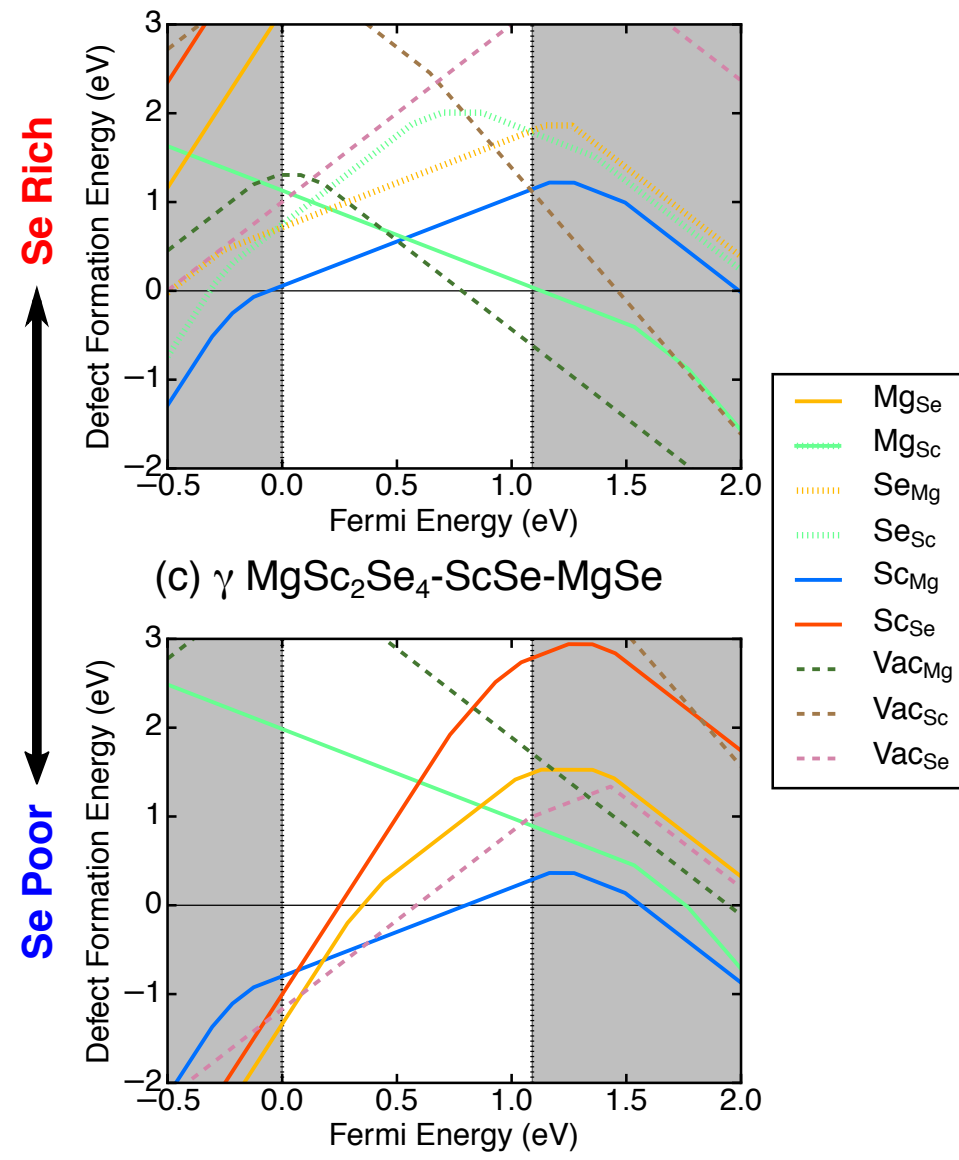

(b) $\beta \mathrm{MgSc}_{2} \mathrm{Se}_{4}-\mathrm{Se}-\mathrm{MgSe}$

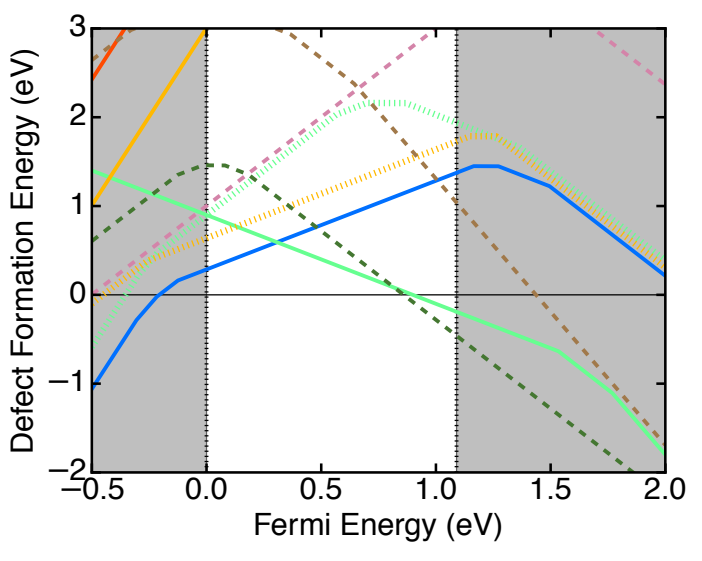

(d) $\delta \mathrm{MgSc}_{2} \mathrm{Se}_{4}-\mathrm{ScSe}_{-} \mathrm{Sc}_{2} \mathrm{Se}_{3}$

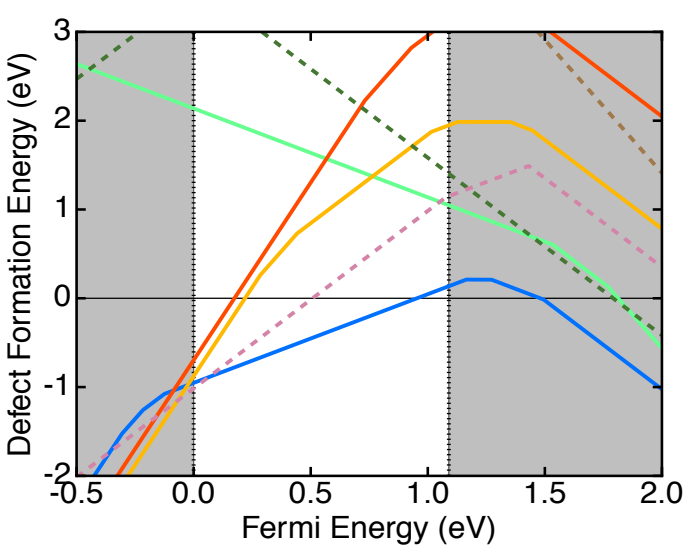

Figure 3: Defect energy $E_{f}\left[X^{q}\right]$ for intrinsic point defects (in Kröger-Vink notation) of $\mathrm{MgSc}_{2} \mathrm{Se}_{4}$ in four facets, $\alpha, \beta, \gamma$ and $\delta$, of the Mg-Sc-Se phase diagram. Regions $\alpha$ and $\beta$ are Se-rich, whereas $\gamma$ and $\delta$ Se-poor. The VBM is arbitrarily set to $0 \mathrm{eV}$ and the white region spans the band-gap $(\sim 1.09 \mathrm{eV})$. Vac in the legend and dashed lines indicate vacancy defects while solid lines correspond to anti-site defects.

each other leading to charge neutrality, the $E_{\text {Fermi }}^{e q}$ is nominally pinned at a Fermi energy where all three defects have similar $E_{f}$. Indeed, a self-consistent calculation of the $E_{F e r m i}^{e q}$ at $300 \mathrm{~K}$ (i.e., assuming defect concentrations equilibrate at $300 \mathrm{~K}$ ) leads to a $E_{F e r m i}=0.46 \mathrm{eV}$ (see Figures 3a and S5a in SI), with defect concentrations of $7.9 \times 10^{11} \mathrm{~cm}^{-3}$ for $\mathrm{Sc}_{\mathrm{Mg}}^{\bullet}, 2.4 \times 10^{11}$ $\mathrm{cm}^{-3}$ for $\mathrm{Mg}_{\mathrm{Sc}}^{\prime}$ and $2.8 \times 10^{11} \mathrm{~cm}^{-3}$ for $\mathrm{Vac}_{\mathrm{Mg}}^{\prime \prime}$. Typically, defect contents above $10^{15} \mathrm{~cm}^{-3}$ are detectable via experiments, such as electron paramagnetic resonance. ${ }^{33,63,64}$ Alternatively, defect concentrations can be expressed in units of per atom or per formula unit. For example, a defect concentration of $10^{15} \mathrm{~cm}^{-3}$ in $\mathrm{MgSc}_{2} \mathrm{Se}_{4}$ corresponds to $\sim 2.4 \times 10^{-8} /$ atom and 
$1.7 \times 10^{-7}$ /(formula unit), respectively. For the $\alpha$-facet of $\mathrm{MgSc}_{2} \mathrm{Se}_{4}$ at $300 \mathrm{~K}$, the Fermi level is "deep" within the band gap, which will lead to low electronic (or hole) conductivity since large thermal energies $\left(\gg k_{B} T\right)$ will be required to ionize free electrons (holes) from the $E_{F e r m i}^{e q}$ into the conduction (valence) band. Qualitatively similar conclusions can be drawn from an analysis of the defects in the $\beta$ facet (Figure 3b and Figure S7a).

However, when defect concentrations are frozen-in from a higher temperature $(\sim 1273 \mathrm{~K}$ used for $\mathrm{MgSc}_{2} \mathrm{Se}_{4}$ synthesis $\left.{ }^{22}\right)$, the $E_{\text {Fermi }}^{\text {frozen }}$ at $300 \mathrm{~K}$ drops below the VBM $(\sim-0.10 \mathrm{eV})$ indicating that the material becomes a $p$-type conductor. Thus, significant hole conductivity can be expected under frozen defect conditions, with free hole concentration of $\sim 2.6 \times 10^{18} \mathrm{~cm}^{-3}$ ( $\sim 0.0001$ per lattice site), which is beyond un-doped semiconductor levels $\left(\sim 10^{10} \mathrm{~cm}^{-3}\right.$ in SI) but below metallic levels ( 1 charge carrier per lattice site). As the temperature at which the defect concentrations are quenched decreases, the $E_{F e r m i}^{\text {frozen }}$ recovers beyond the VBM and reaches $\sim 0.02 \mathrm{eV}$ at $800 \mathrm{~K}$ (Figure S7a), indicating the importance of slow cooling conditions to reduce hole conductivity during the synthesis of $\mathrm{MgSc}_{2} \mathrm{Se}_{4}$.

\section{Se-poor domain, $\gamma \mathrm{MgSc}_{2} \mathrm{Se}_{4}$-ScSe-MgSe}

The Se-poor region (Figure 3c) is dominated by $n$-type defects, such as $\mathrm{Sc}_{\mathrm{Mg}}^{\bullet}$ (dark blue), $\operatorname{Vac}_{\mathrm{Se}}^{\bullet \bullet}$ (dashed red), and $\mathrm{Mg}_{\mathrm{Se}}^{\bullet \bullet \bullet}$ (orange). Although the formation energies of a few defects are negative across the band gap, as in the case of $\mathrm{Sc}_{\mathrm{Mg}}^{\bullet}$ for $E_{F e r m i}<0.7 \mathrm{eV}$ (Figure 3c), the spontaneous formation of such charged defects is constrained by the condition of charge neutrality in $\mathrm{MgSc}_{2} \mathrm{Se}_{4}$.

The self-consistent equilibrium Fermi level $(\sim 1.08 \mathrm{eV}$ at $300 \mathrm{~K})$ for the Se-poor region is mainly set by the $\mathrm{Sc}_{\mathrm{Mg}}^{\bullet}$ defect. However for temperatures above $300 \mathrm{~K}$, the $E_{F e r m i}^{e q}$ exceeds the Conduction Band Minimum (CBM, $~ 1.1 \mathrm{eV}$, Figure S7a), suggesting the occurrence of spontaneous electronic conductivity when the spinel is synthesized under Se-poor conditions. Furthermore, when defect concentrations are frozen-in from $\sim 1273 \mathrm{~K}, E_{\text {Fermi }}^{\text {frozen }}$ is well above the $\mathrm{CBM}(\sim 1.4 \mathrm{eV})$ at $300 \mathrm{~K}$, suggesting that fast cooling during synthesis will likely 
increase the electronic conductivity. Similar conclusions can be extended by evaluating the defect energies in the $\delta$ facet (Figure 3d, and Figure S7a in the SI), where the equilibrium Fermi level is beyond the CBM even at $300 \mathrm{~K}$, suggesting that preventing intrinsic electronic conductivity in $\mathrm{MgSc}_{2} \mathrm{Se}_{4}$ in Se-poor conditions may be challenging.

\section{$4.2 \quad \operatorname{MgIn}_{2} \mathrm{~S}_{4}$}

Figure 4 plots the defect formation energies for the S-rich $(\alpha)$ and S-poor $(\gamma)$ domains as a function of Fermi energy in the $\mathrm{MgIn}_{2} \mathrm{~S}_{4}$ spinel. Analogous to $\mathrm{MgSc}_{2} \mathrm{Se}_{4}$ (Figure 3a), the stable defects in the S-rich domain of $\mathrm{MgIn}_{2} \mathrm{~S}_{4}$ (Figure 4a), include the $n$-type $\operatorname{In}_{\mathrm{Mg}}^{\bullet}$ (solid blue), and the $p$-type $\mathrm{Mg}_{\mathrm{In}}^{\prime}$ (light green) and $\mathrm{Vac}_{\mathrm{Mg}}^{\prime \prime}$ (dashed green). For the $\alpha$-facet (S-rich domain), the resulting $E_{F e r m i}^{e q}$ is $\sim 0.88 \mathrm{eV}$ at $300 \mathrm{~K}$, corresponding to a free hole concentration of $\sim 6.46 \times 10^{4} \mathrm{~cm}^{-3}$. Other notable defects, such as $\mathrm{Vac}_{\mathrm{In}}^{\prime \prime \prime}$, Vac $\mathrm{Va}_{\mathrm{S}}^{\bullet \bullet}$ and $\mathrm{S}_{\mathrm{In}}^{\prime}$ are not expected to play a dominant role in $\operatorname{MgIn}_{2} \mathrm{~S}_{4}$.

Since the equilibrium Fermi energy is pinned near the middle of the band-gap $(\sim 0.88 \mathrm{eV}$ Figure 4a) by self-compensating charged defects, the material will exhibit low electronic conductivity under equilibrium S-rich conditions. Nevertheless, the equilibrium defect concentrations are significant, $\sim 4.9 \times 10^{17} \mathrm{~cm}^{-3}$ for $\operatorname{In}_{\mathrm{Mg}}^{\bullet}, 4.9 \times 10^{17} \mathrm{~cm}^{-3} \mathrm{Mg}_{\mathrm{In}}^{\prime}$ and $7.8 \times 10^{13} \mathrm{~cm}^{-3}$ $\mathrm{Vac}_{\mathrm{Mg}}^{\prime \prime}$. Such high concentrations of anti-site $\mathrm{In}_{\mathrm{Mg}}^{\bullet}$ and $\mathrm{Mg}_{\mathrm{In}}^{\prime}$ defects indicate that the spinel undergoes a high degree of "inversion" — Mg and In exchanging their lattice sites ${ }^{65}$ — besides exhibiting significant $\mathrm{Mg}$ vacancies during S-rich synthesis conditions.

The analysis of the defect formation energies in the S-poor facet ( $\gamma$, Figure 4b) suggests that the $\operatorname{Vac}_{\mathrm{S}}^{\bullet}$ and $\operatorname{In}_{\mathrm{S}}^{\bullet \bullet}$ defects can influence $E_{F e r m i}^{e q}$, apart from the $\mathrm{In}_{\mathrm{Mg}}^{\bullet}, \mathrm{Mg}_{\mathrm{In}}^{\prime}$ and $\mathrm{Vac}_{\mathrm{Mg}}^{\prime \prime}$. Interestingly, the self-consistent Fermi level of the $\gamma$ facet is $\sim 1.53 \mathrm{eV}$, which corresponds approximatively to charge-compensation between the $\operatorname{In}_{\mathrm{Mg}}^{\bullet}$ and $\mathrm{Mg}_{\mathrm{In}}^{\prime}$ defects. Furthermore, the equilibrium defect concentrations $\left(\sim 4.9 \times 10^{17}\right.$ for both $\operatorname{In}_{\mathrm{Mg}}^{\bullet}$ and $\mathrm{Mg}_{\mathrm{In}}^{\prime}$, respectively $)$ estimated under S-poor conditions compare well with a S-rich environment, indicating that the $\operatorname{MgIn}_{2} \mathrm{~S}_{4}$ is expected to undergo a substantial degree of spinel inversion irrespective of 
(a) $\alpha M g \ln _{2} S_{4}-S-\ln _{2} S_{3}$

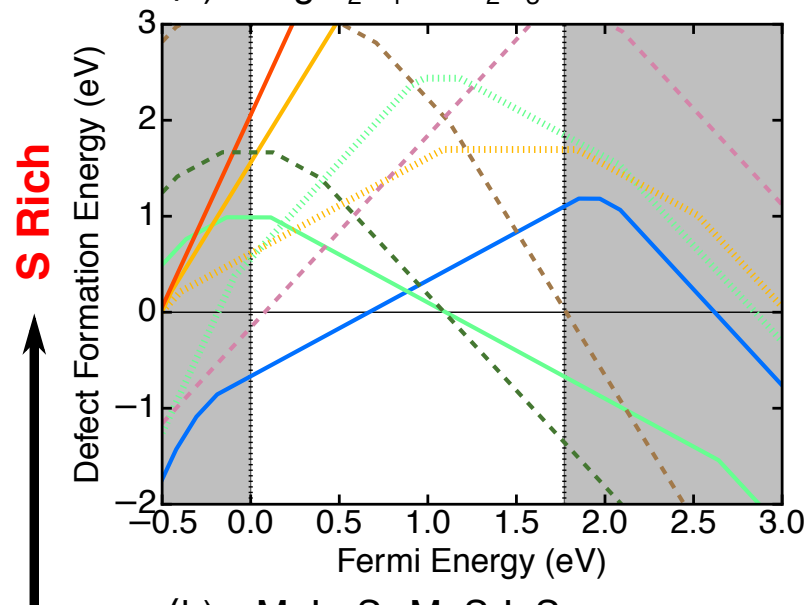

(b) $\gamma \mathrm{MgIn}_{2} \mathrm{~S}_{4}-\mathrm{MgS}-\operatorname{InS}$

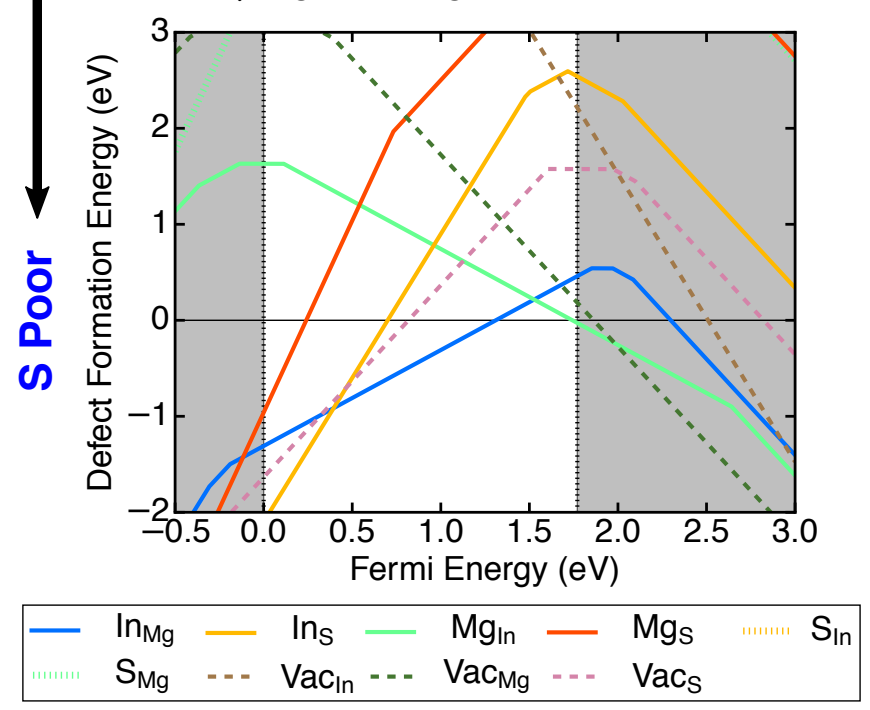

Figure 4: (Colors online) $E_{f}\left[X^{q}\right]$ for intrinsic point defects (in Kröger-Vink notation) of $\mathrm{MgIn}_{2} \mathrm{~S}_{4}$ for two regions of the phase diagram ( $\alpha$ and $\gamma$, refer to Figure 3). Region $\alpha$ is $\mathrm{S}$-rich, whereas $\gamma$ is S-poor. The VBM is set to $0 \mathrm{eV}$ and the white region is the bandgap $(\sim 1.77 \mathrm{eV})$. Vac in legend and dashed lines indicate vacancy defects while solid lines correspond to anti-site defects.

synthesis conditions, in agreement with experimental observations. ${ }^{65}$

Similar to $\mathrm{MgSc}_{2} \mathrm{Se}_{4}$, cooling rates during synthesis are expected to play a major role in determining the intrinsic hole/electronic conductivity in the In-spinel (Figure S7b). For example, under frozen-in defect concentrations from $\sim 1273 \mathrm{~K}$, the $E_{F e r m i}^{\text {frozen }}$ and $c[e / h]^{\text {frozen }}$ are $\sim 0.10 \mathrm{eV}, 1.17 \times 10^{18}$ (free holes) for S-rich and $\sim 1.80 \mathrm{eV}(>\mathrm{CBM}), 1.12 \times 10^{19}$ (free electrons) for S-poor, respectively. $\operatorname{MgIn}_{2} \mathrm{~S}_{4}$ is expected to exhibit $p$-type and $n$-type conduc- 
tivity in S-rich and S-poor conditions, respectively, under quenched defect concentrations. Thus, the protocols to synthesize $\mathrm{MgIn}_{2} \mathrm{~S}_{4}$ requires careful tuning to allow for slow cooling and S-rich conditions.

\section{$4.3 \quad \mathrm{MgSc}_{2} \mathrm{~S}_{4}$}

Figure 5 shows the formation energies of native defects of $\mathrm{MgSc}_{2} \mathrm{~S}_{4}$ in both $\mathrm{S}$-rich ( $\alpha$ facet) and S-poor ( $\gamma$ facet) domains. The defect energetics in $\mathrm{MgSc}_{2} \mathrm{~S}_{4}$ are similar to the Se-spinel, in the anion-rich domain (Figure 5a), with the dominant defects being $\mathrm{Sc}_{\mathrm{Mg}}^{\bullet}, \mathrm{Mg}_{\mathrm{Sc}}^{\prime}$, and $\operatorname{Vac}_{\mathrm{Mg}}^{\prime \prime}$. The $E_{F e r m i}^{e q}$, calculated self-consistently at $300 \mathrm{~K}$ is $\sim 0.4 \mathrm{eV}$ and roughly corresponds to the self-compensation of $\mathrm{Vac}_{\mathrm{Mg}}^{\prime \prime}$ and $\mathrm{Mg}_{\mathrm{Sc}}^{\prime}$ with $\mathrm{Sc}_{\mathrm{Mg}}^{\bullet}$. Therefore, when $\mathrm{MgSc}_{2} \mathrm{~S}_{4}$ is prepared under S-rich conditions it should exhibit a small degree of spinel inversion $\left(\mathrm{Sc}_{\mathrm{Mg}}^{\bullet} 1.7 \times 10^{11}\right.$ $\mathrm{cm}^{-3}$ and $\left.\mathrm{Mg}_{\mathrm{Sc}}^{\prime} \sim 3.7 \times 10^{11} \mathrm{~cm}^{-3}\right)$, and low hole conductivity $\left(c[h]^{e q} \sim 2.01 \times 10^{11} \mathrm{~cm}^{-3}\right)$. Also, under frozen-in defect conditions (from $1273 \mathrm{~K}$ ), $\mathrm{MgSc}_{2} \mathrm{~S}_{4}$ becomes a spontaneous $p$ type conductor similar to $\mathrm{MgSc}_{2} \mathrm{Se}_{4}$, with $E_{\text {Fermi }}^{\text {frozen }}$ drifting below the $\mathrm{VBM}(\sim-0.06 \mathrm{eV})$ resulting in a larger $c[h]^{\text {frozen }} \sim 1.18 \times 10^{19} \mathrm{~cm}^{-3}$.

The dominant point defects in the S-poor region $(\gamma$, Figure $5 \mathrm{~b})$ are $\mathrm{Mg}_{\mathrm{S}}^{\bullet \bullet}, \mathrm{Sc}_{\mathrm{Mg}}^{\bullet}, \mathrm{Vac}_{\mathrm{S}}^{\bullet \bullet}$ and $\mathrm{Mg}_{\mathrm{Sc}}^{\prime}$, with $\operatorname{Vac}_{\mathrm{S}}^{\bullet \bullet}$ displaying the lowest $E_{f}\left[X^{q}\right]$ across the band gap, up to $E_{F e r m i} \sim 1.5 \mathrm{eV}$. In the $\gamma$ (and $\delta$, Figure S7c) facet, the equilibrium Fermi level at $300 \mathrm{~K}$ is beyond the CBM, indicating spontaneous electronic conductivity under S-poor conditions. Under quenched defect conditions (from $1273 \mathrm{~K}$ ), $E_{\text {Fermi }}^{\text {frozen }}$ is found to be deeper into the conduction band $(\sim 1.80 \mathrm{eV})$ compared to equilibrium at $300 \mathrm{~K}$ (Figure S7c). Indeed, $c[e]^{e q}$ is estimated to be $\sim 1.8 \times 10^{15} \mathrm{~cm}^{-3}$ under equilibrium at $300 \mathrm{~K}$, while the concentration increases by nearly 5 orders of magnitude $\left(c[e]^{\text {frozen }} \sim 7.8 \times 10^{19} \mathrm{~cm}^{-3}\right)$ under quenched conditions. Thus, suppressing intrinsic electronic conductivity in $\mathrm{MgSc}_{2} \mathrm{~S}_{4}$ under S-poor conditions represents a major challenge. 
(a) $\alpha \mathrm{MgSc}_{2} \mathrm{~S}_{4}-\mathrm{S}_{-} \mathrm{Sc}_{2} \mathrm{~S}_{3}$

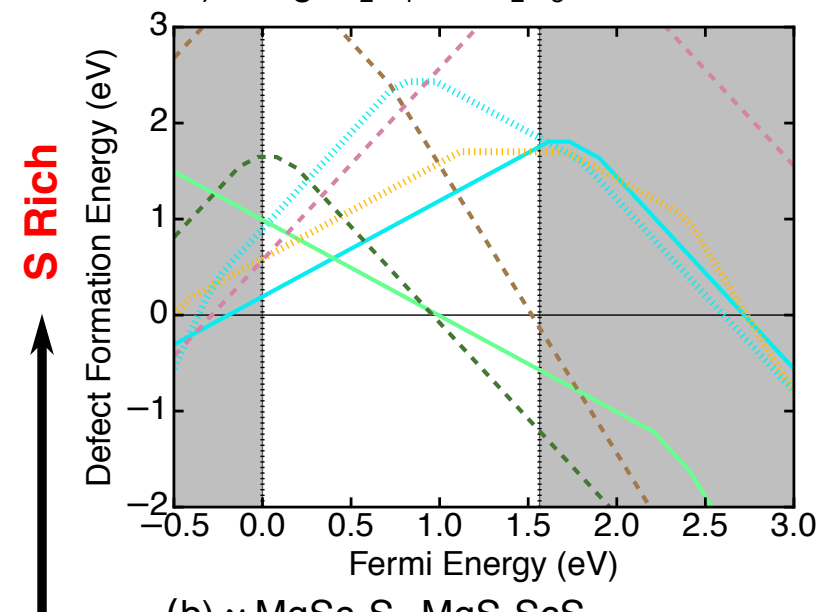

(b) $\gamma \mathrm{MgSc}_{2} \mathrm{~S}_{4}$-MgS-ScS

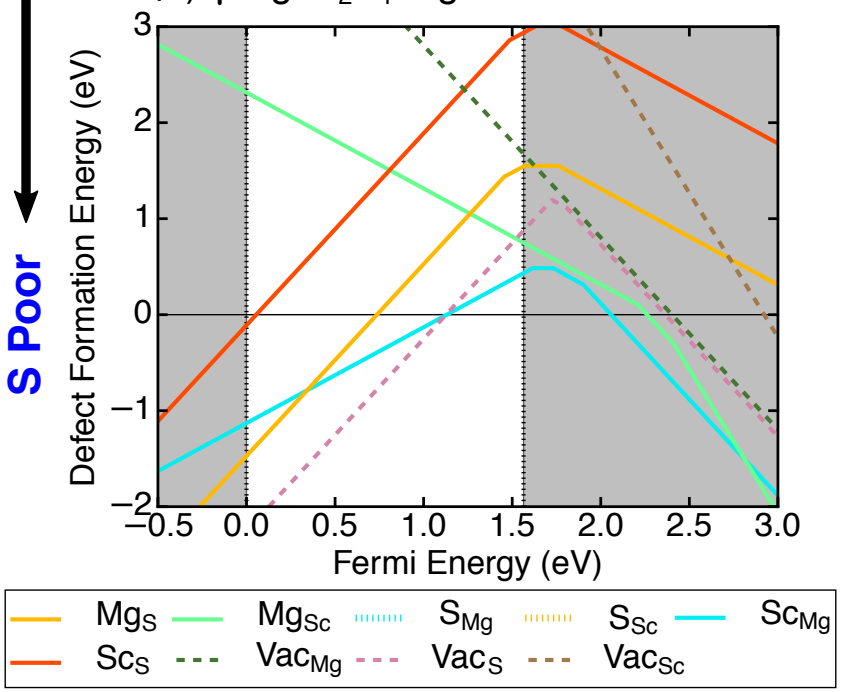

Figure 5: $E_{f}\left[X^{q}\right]$ for intrinsic point defects (in Kröger-Vink notation) of $\mathrm{MgSc}_{2} \mathrm{~S}_{4}$ for two regions of the phase diagram ( $\alpha$ and $\gamma$, refer to Figure 3). Region $\alpha$ is S-rich, whereas $\gamma$ is $\mathrm{S}$-poor. The VBM is set to $0 \mathrm{eV}$ and the white region is the band-gap $(\sim 1.56 \mathrm{eV})$. Vac in legend and dashed lines indicate vacancy defects and solid lines indicate anti-site defects.

\section{$5 \quad$ Extrinsic defects in $\mathrm{MgSc}_{2} \mathrm{Se}_{4}$}

High ionic conductivity in materials is often achieved if the concentration of mobile vacancies is increased. One strategy commonly adopted to increase ionic conductivity in solid electrolytes is extrinsic doping, specifically doping the anion sub-lattice. ${ }^{66-68}$ Nominally, the selection of an extrinsic dopant follows the rule of thumb of finding similar-sized cations (anions) for aliovalent substitution in the lattice. In addition, it is desirable that the substituting element is not redox-active, which will minimize the occurrence of redox side-reactions 
in ionic conductors.

In the case of the spinel Mg-conductors discussed in this work, the electronic conductivity primarily arises from anti-site defects, such as $\mathrm{Sc}_{\mathrm{Mg}}^{\bullet}$ and $\mathrm{In}_{\mathrm{Mg}}^{\bullet}$, pushing the equilibrium Fermi level close (or beyond) the CBM level at $300 \mathrm{~K}$. A pathway to curb the formation of anti-site defects is doping the metal site (Sc or In) with cations that are less likely to promote spinel inversion. For example, metal ions with higher oxidations states (such as $\mathrm{Zr}^{4+}$ and $\mathrm{Nb}^{5+}$ ) or those manifesting a stronger octahedral site preference than $\mathrm{Mg}^{2+}$, are less likely to occupy the tetrahedral spinel sites. ${ }^{69}$ Thus, cation doping on the Sc site can inhibit the formation of anti-site $\mathrm{Sc}_{\mathrm{Mg}}$ defects in $\mathrm{MgSc}_{2} \mathrm{Se}_{4}$ (and $\mathrm{MgSc}_{2} \mathrm{~S}_{4}$ ).

Figure 6 plots the defect formation energies for extrinsic doping of several non-redox tetravalent (Ce, Ge, Sn, Pb, Ti and Zr, solid lines in Figure 6a), and pentavalent (As, Bi, $\mathrm{Na}$ and $\mathrm{Ta}$, dashed lines) cations on $\mathrm{Sc}$ in $\mathrm{MgSc}_{2} \mathrm{Se}_{4}$, as well as anion doping on $\mathrm{Se}^{2-}$ with monovalent anions $\left(\mathrm{Cl}^{-}, \mathrm{Br}^{-}\right.$and $\mathrm{I}^{-}$, Figure $\left.6 \mathrm{~b}\right)$. Because of the Se-poor synthesis conditions normally encountered, we restrict the analysis only to the $\gamma$ facet $\left(\mathrm{MgSc}_{2} \mathrm{Se}_{4}-\mathrm{MgSe}-\mathrm{ScSe}\right)$, while calculations of the $\alpha, \beta$ and $\delta$ facets of $\mathrm{MgSc}_{2} \mathrm{Se}_{4}$ are discussed in Figures $\mathrm{S} 7$ and $\mathrm{S} 8$ of the SI. Note that the chemical potential of each extrinsic dopant in Eq. 1 is set by the most stable phase in the Mg-Sc-Se-[extrinsic dopant] phase diagram that is in equilibrium with $\mathrm{MgSc}_{2} \mathrm{Se}_{4}, \mathrm{MgSe}$, and ScSe (accessed via the Materials Project ${ }^{56}$ ). For example, in the case of $\mathrm{Cl}^{-}$doping on $\mathrm{Se}^{2-}, \mu_{\mathrm{Cl}}$ is determined by the facet $\mathrm{MgSc}_{2} \mathrm{Se}_{4}-\mathrm{MgSe}-\mathrm{ScSe}-\mathrm{MgCl}_{2}$.

Data in Figure 6 suggests that extrinsic doping of several cations, such as Bi, Nb, Ta, $\mathrm{Ge}$, Sn and $\mathrm{Pb}$ on $\mathrm{Sc}$ in $\mathrm{MgSc}_{2} \mathrm{Se}_{4}$ is highly unfavourable (with $E_{f}\left[X^{q}\right] \geq 1 \mathrm{eV}$ ). In contrast, halogen doping on Se (Figure 6b), Ce (solid green line in Figure 6a), Ti (solid light blue), and $\mathrm{Zr}$ (solid red line) doping on Sc appear favorable. Specifically, halogen substitution on Se and $\mathrm{Zr}_{\mathrm{Sc}}^{\bullet}$ show a negative formation energy over a wide portion of the band-gap.

Since the behavior of halogen doping (and $\mathrm{Zr}_{\mathrm{Sc}}^{\bullet}$ ) is similar to intrinsic $n$-type $\mathrm{Sc}_{\mathrm{Mg}}^{\bullet}$ (Figure 3c) anti-sites, anion (and $\mathrm{Zr}$ ) doping in $\mathrm{MgSc}_{2} \mathrm{Se}_{4}$ may not be beneficial since the $E_{F e r m i}^{e q}$ is likely to be pushed into the conduction band at $300 \mathrm{~K}$. However, $n$-type $\mathrm{Ce}_{\mathbf{S c}_{\mathrm{c}}}$ and $\mathrm{Ti}_{\mathrm{Sc}}^{\bullet}$ 
(a) Cation doping $\mathrm{MgSc}_{2} \mathrm{Se}_{4}-\mathrm{MgSe}-\mathrm{ScSe}$

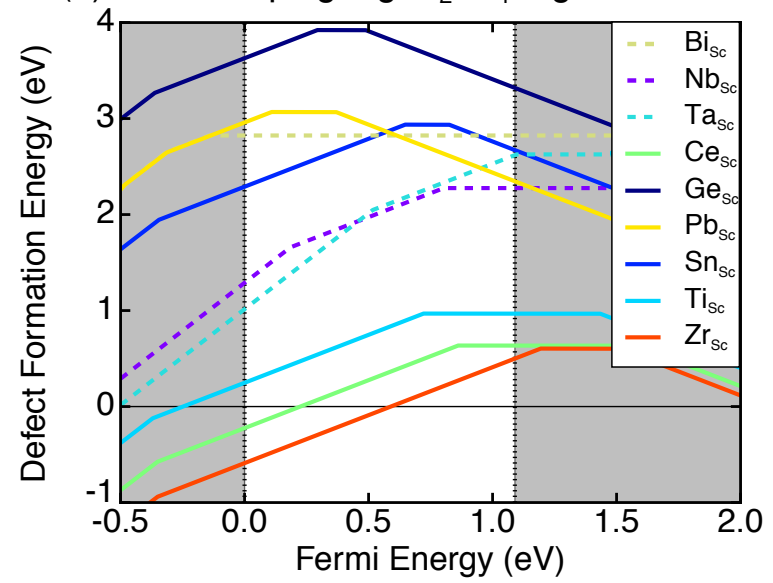

(b) Anion doping $\mathrm{MgSc}_{2} \mathrm{Se}_{4}-\mathrm{MgSe}-\mathrm{ScSe}$

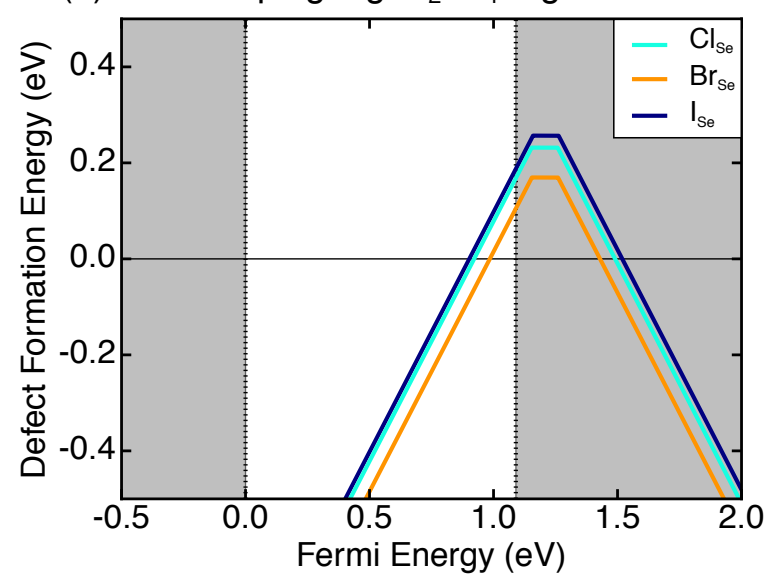

Figure 6: $\quad E_{f}\left[X^{q}\right]$ for extrinsic cation (a) and anion (b) substitution in $\mathrm{MgSc}_{2} \mathrm{Se}_{4}$ under Se-poor conditions $(\gamma$-facet). In panel (a), tetravalent and pentavalent cations are shown by solid and dashed lines, respectively. The $\mathrm{VBM}$ is set to $0 \mathrm{eV}$ and the white region is the band-gap $(\sim 1.09 \mathrm{eV}) . E_{f}\left[X^{q}\right]$ is not shown for $\mathrm{As}_{\mathrm{Sc}}$ substitution, since the values are above $4 \mathrm{eV}$ through the Fermi energy range considered.

show fairly deep donor transition levels away from the CBM (Figure 6a). Indeed, $E_{F e r m i}^{e q}$ at $300 \mathrm{~K}$ for the $\mathrm{Ce}_{\mathrm{Sc}}^{\bullet}$ and $\mathrm{Ti}_{\mathrm{Sc}}^{\bullet}$, without considering the presence of intrinsic anti-sites, are $\sim 0.82 \mathrm{eV}$ and $0.58 \mathrm{eV}$, respectively. Thus, Ce and Ti doping should not increase the electronic conductivity of $\mathrm{MgSc}_{2} \mathrm{Se}_{4}$, though their effect on the $\mathrm{Sc}_{\mathrm{Mg}}$ formation energies requires more investigation. 


\section{Discussion}

Using first-principles defect energy calculations, we analyzed the defect chemistry in chalcogenide $\mathrm{Mg}$ spinels, namely $\mathrm{MgSc}_{2} \mathrm{Se}_{4}, \mathrm{MgSc}_{2} \mathrm{~S}_{4}$ and $\mathrm{MgIn}_{2} \mathrm{~S}_{4}$, and have summarized the Fermi energies and free-carrier concentrations in Table 1 (defect concentrations are also tabulated in Table S1) for a representative anion-rich and anion-poor equilibrium. Under all conditions, anti-sites $\left(\mathrm{Mg}_{\{\mathrm{Sc} / \mathrm{In}\}}\right.$ and $\left.\{\mathrm{Sc} / \mathrm{In}\}_{\mathrm{Mg}}\right)$ and $\mathrm{Mg}$-vacancies are the dominant defects, while anion vacancies only show up for $\mathrm{MgSc}_{2} \mathrm{~S}_{4}$ under S-poor conditions.

Table 1: Defect energetics in the $\mathrm{MgA}_{2} \mathrm{Z}_{4}$ spinels $(\mathrm{A}=\mathrm{Sc}, \mathrm{In}, \mathrm{Z}=\mathrm{S}, \mathrm{Se})$, for both anionrich $(\alpha)$ and anion-poor $(\gamma)$ conditions (facets). Self-consistent $E_{F e r m i}^{e q}$ at $300 \mathrm{~K}$ (in eV) and Fermi levels with quenched defect content (from $1273 \mathrm{~K}, E_{\mathrm{Fermi}}^{\text {frozen }}$ ), are indicated. $c[e / h]^{e q}$ and $c[e / h]^{\text {frozen }}$ (in $\mathrm{cm}^{-3}$ at $300 \mathrm{~K}$ ) are the free charge-carrier concentration in the self-consistent equilibrium and frozen defect scenarios, with $e$ and $h$ for electrons and holes. The charge of the dominant defect is indicated with respect to the charged state of the defect at $E_{\text {Fermi }}^{e q}$.

\begin{tabular}{|c|c|c|c|c|c|c|}
\hline Condition & Dominant defects & Carrier & $E_{\text {Fermi }}^{e q}$ & $c[e / h]^{e q}$ & $E_{\text {Fermi }}^{\text {frozen }}$ & $c[e / h]^{\text {frozen }}$ \\
\hline \multicolumn{7}{|c|}{$\mathrm{MgSc}_{2} \mathrm{Se}_{4}\left(\mathrm{E}_{\text {gap }}=1.09 \mathrm{eV}\right)$} \\
\hline Se-rich $(\alpha)$ & $\mathrm{Sc}_{\mathrm{Mg}}^{\bullet}, \mathrm{Mg}_{\mathrm{Sc}}^{\prime}, \mathrm{Vac}_{\mathrm{Mg}}^{\prime \prime}$ & $h^{+}$ & 0.46 & $8.42 \times 10^{8}$ & -0.10 & $2.58 \times 10^{18}$ \\
\hline Se-poor $(\gamma)$ & $\mathrm{Sc}_{\mathrm{Mg}}^{\bullet}$ & $e^{-}$ & 1.08 & $7.96 \times 10^{15}$ & 1.39 & $2.77 \times 10^{19}$ \\
\hline \multicolumn{7}{|c|}{$\operatorname{MgIn}_{2} S_{4}\left(E_{g a p}=1.77 \mathrm{eV}\right)$} \\
\hline S-rich $(\alpha)$ & $\mathrm{In}_{\mathrm{Mr}}^{\bullet}, \mathrm{Mg}_{\mathrm{In}}^{\prime}, \mathrm{Vac}_{\mathrm{Mr}}^{\prime \prime}$ & $h^{+}$ & 0.88 & $6.46 \times 10^{4}$ & 0.10 & $1.17 \times 10^{18}$ \\
\hline S-poor $(\gamma)$ & $\operatorname{In}_{\mathrm{Mg}}^{\bullet}, \mathrm{Mg}_{\mathrm{In}}^{\prime}, \mathrm{Vac}_{\mathrm{Mg}}^{\prime \prime \prime}$ & $e^{-}$ & 1.53 & $4.10 \times 10^{14}$ & 1.80 & $1.12 \times 10^{19}$ \\
\hline \multicolumn{7}{|c|}{$\mathrm{MgSc}_{2} \mathrm{~S}_{4}\left(\mathrm{E}_{q a p}=1.55 \mathrm{eV}\right)$} \\
\hline S-rich $(\alpha)$ & $\mathrm{Sc}_{\mathrm{M} \alpha}^{\bullet}, \mathrm{Mg}_{\mathrm{S} c}^{\prime}, \mathrm{Vac}_{\mathrm{Mr}}^{\prime \prime}$ & $h^{+}$ & 0.40 & $2.01 \times 10^{11}$ & -0.06 & $1.18 \times 10^{19}$ \\
\hline S-poor $(\gamma)$ & $\mathrm{Sc}_{\mathrm{Mg}}^{\bullet}, \mathrm{Mg}_{\mathrm{Sc}}^{\prime}, \mathrm{Vac}_{\mathrm{S}}^{\bullet \bullet}$ & $e^{-}$ & 1.48 & $1.81 \times 10^{15}$ & 1.80 & $7.86 \times 10^{19}$ \\
\hline
\end{tabular}

\subsection{Anion-rich vs. anion-poor conditions}

All three spinels display markedly different defect energetics in the anion (S/Se)-rich and anion-poor domains, under equilibrium defect concentrations. In the case of anion-rich conditions ( $\alpha$ facet), the spinels exhibit marginal $p$-type behavior with low carrier concentrations, due to the presence of charged anti-sites $\left(\{\mathrm{Sc} / \mathrm{In}\}_{\mathrm{Mg}}^{\bullet}\right.$ and $\left.\mathrm{Mg}_{\{\mathrm{Sc} / \mathrm{In}\}}^{\prime}\right)$, and $\operatorname{Vac}_{\mathrm{Mg}}^{\prime \prime}$, which charge-compensate each other and pin the $E_{F e r m i}^{e q}$ within the respective band gaps. Since the $E_{F e r m i}^{e q}$ is far away from the VBM (or CBM), i.e., $\gg k_{B} T$, the hole (or electronic) 
conductivity is not expected to be significant (see Table 1). Hence, the synthesis of the chalocogenide conductors in anion-rich environments should curtail, to a large extent, the undesired hole/electron conductivity for application as a Mg solid electrolyte. However, synthesis of the Se spinels requires high temperatures $\left(>1000{ }^{\circ} \mathrm{C}\right),{ }^{12,13,22}$ at which elemental Se (b.p. $\left.\sim 685^{\circ} \mathrm{C}\right)$ and $\mathrm{S}\left(\sim 444^{\circ} \mathrm{C}\right)$ vaporize and may lead to anion-poor conditions. One potential strategy to mitigate anion loss during synthesis is to use the respective stoichiometric binaries, such as $\mathrm{MgSe}$ and $\mathrm{Sc}_{2} \mathrm{Se}_{3}$ to form $\mathrm{MgSc}_{2} \mathrm{Se}_{4}$, at high temperature. ${ }^{12,13}$

Unlike anion-rich conditions, the dominant $n$-type $\mathrm{Sc}_{\mathrm{Mg}}^{\bullet}$ anti-sites in the anion poor domain ( $\gamma$ facet) push the $E_{F e r m i}^{e q}$ beyond the CBM in both Sc-spinels, ensuring spontaneous electronic conductivity. We speculate that the low $\mathrm{Sc}_{\mathrm{Mg}}^{\bullet}$ equilibrium concentration $\left(\sim 7.96 \times 10^{15} \mathrm{~cm}^{-3}\right.$, Table S1) in $\mathrm{MgSc}_{2} \mathrm{Se}_{4}$ may not significantly affect the XRD pattern ${ }^{22}$ with respect to an ideal spinel structure and might be hard to detect using bulk characterization experiments. Also, the $c[e]^{e q} \sim 7.96 \times 10^{15}$ in $\mathrm{MgSc}_{2} \mathrm{Se}_{4}$ (Table 1), corresponding to $\sim 0.0001$ free electrons per lattice site, is remarkably high compared to the intrinsic carrier concentration of typical semi-conductors (e.g., $\sim 10^{10} \mathrm{~cm}^{-3}$ in $\mathrm{Si}$ ), but significantly below metallic levels $\left(\sim 1 \mathrm{e}^{-}\right.$per lattice site). Although the $\mathrm{In}_{\mathrm{Mg}}^{\bullet}$ and $\mathrm{Mg}_{\mathrm{In}}^{\prime}$ defects chargecompensate in $\mathrm{MgIn}_{2} \mathrm{~S}_{4}$, the $E_{\text {Fermi }}^{e q}$ under S-poor conditions is only $\sim 0.2 \mathrm{eV}$ below the $\mathrm{CBM}$, indicating significant $n$-type conductivity. Indeed, a previous measurement of the Hall effect in $\operatorname{MgIn}_{2} \mathrm{~S}_{4}{ }^{65}$ reported a moderate resistivity of $\sim 8.2 \times 10^{3} \Omega / \mathrm{cm}$ and a free-

electron concentration of $\sim 6.4 \times 10^{15} \mathrm{~cm}^{-3}$, in reasonable agreement with our $c[e]^{e q}$ estimate of $\sim 4.1 \times 10^{14} \mathrm{~cm}^{-3}$ in the $\gamma$ facet.

\subsection{Impact of cooling rates}

The variation of $c[e / h]^{\text {frozen }}$ and $E_{F e r m i}^{\text {frozen }}$ as a function of quench temperature - the temperature at which the defect concentrations are frozen - is plotted in Figures 7 and S7, respectively. Solid and dashed lines in Figure 7 correspond to anion-rich and anion-poor conditions, while the blue, red, and green colors indicate $\mathrm{MgSc}_{2} \mathrm{Se}_{4}, \mathrm{MgIn}_{2} \mathrm{~S}_{4}$, and $\mathrm{MgSc}_{2} \mathrm{~S}_{4}$. 
The quench temperature, which is determined by the cooling rate significantly impacts the hole/electron conductivity. For example, all three spinels are expected to show spontaneous $h^{+}$conductivity at $300 \mathrm{~K}$ in the anion-rich domain ( $\alpha$ facet) when defect concentrations are quenched from $1300 \mathrm{~K}$, contrary to the equilibrium scenario which would give negligible $p / n$-type conduction, as indicated by Figure 7 and Table 1. Furthermore, quenched defect conditions in the anion-poor domain ( $\gamma$ facet) dramatically increase the $n$-type conductivity

in all spinels, resulting in $c[e]^{\text {frozen }}$ that are $\approx 3$ to 4 orders of magnitude higher than $c[e]^{e q}$ (Table 1, Figure 7). As a result, the synthesis of the chalcogenide spinels discussed in this work not only requires anion-rich conditions but also slow cooling post-synthesis (i.e., low quench temperatures, $\sim 400-500 \mathrm{~K}$, see Figure S7) to minimize the electronic conductivity.

\subsection{Inversion in $\operatorname{MgIn}_{2} \mathrm{~S}_{4}$}

In comparison to the Sc-compounds, the defect energies in $\operatorname{MgIn}_{2} \mathrm{~S}_{4}$ (Figure $4 \mathrm{a}$ and b) dictate that under equilibrium, the $E_{F e r m i}^{e q}$ should be largely set by charge-compensating $\operatorname{In}_{\mathrm{Mg}}^{\bullet}, \mathrm{Mg}_{\mathrm{In}}^{\prime}$, and $\mathrm{Vac}_{\mathrm{Mg}}^{\prime \prime}$, corresponding to a lower hole/electron conductivity in either S-rich or S-poor condition. Notably, the combination of $\mathrm{In}_{\mathrm{Mg}}^{\bullet}$ and $\mathrm{Mg}_{\mathrm{In}}^{\prime}$ anti-sites leads to inversion in the spinel (i.e., $\mathrm{Mg}$ and In exchange their respective sites), resulting in a $\left[\mathrm{Mg}_{1-i} \mathrm{In}_{i}\right]\left[\mathrm{Mg}_{i} \operatorname{In}_{2-i}\right] \mathrm{S}_{4}$ stoichiometry, where $i$ is the degree of inversion. Our calculations indicate that $\mathrm{MgIn}_{2} \mathrm{~S}_{4}$ will display significant spinel inversion under both S-rich and S-poor equilibrium conditions, with expected concentrations of $4.9 \times 10^{17}$ for both $\mathrm{In}_{\mathrm{Mg}}^{\bullet}$ and $\mathrm{Mg}_{\mathrm{In}}^{\prime}$ (Table S1), which qualitatively agrees with experimental reports. ${ }^{65,70-74}$ Spinel inversion can impact Mg-mobility and in turn the overall ionic conductivity since inverted structures will possess multiple local $\mathrm{Mg}-$ In configurations.

Interestingly, the Sc-containing spinels are not expected to invert as much as the $\mathrm{MgIn}_{2} \mathrm{~S}_{4}$. For example, $\mathrm{MgSc}_{2} \mathrm{~S}_{4}$ exhibits fewer anti-sites $\left(\mathrm{Sc}_{\mathrm{Mg}}^{\bullet}, \mathrm{Mg}_{\mathrm{Sc}}^{\prime} \sim 10^{11} \mathrm{~cm}^{-3}\right)$ than $\operatorname{MgIn}_{2} \mathrm{~S}_{4}$ under S-rich equilibrium conditions (Table S1). The tendency of $\mathrm{MgIn}_{2} \mathrm{~S}_{4}$ to invert readily may be due to the $s p^{3}$ hybridization in the tetrahedra that is better accommodated by In 


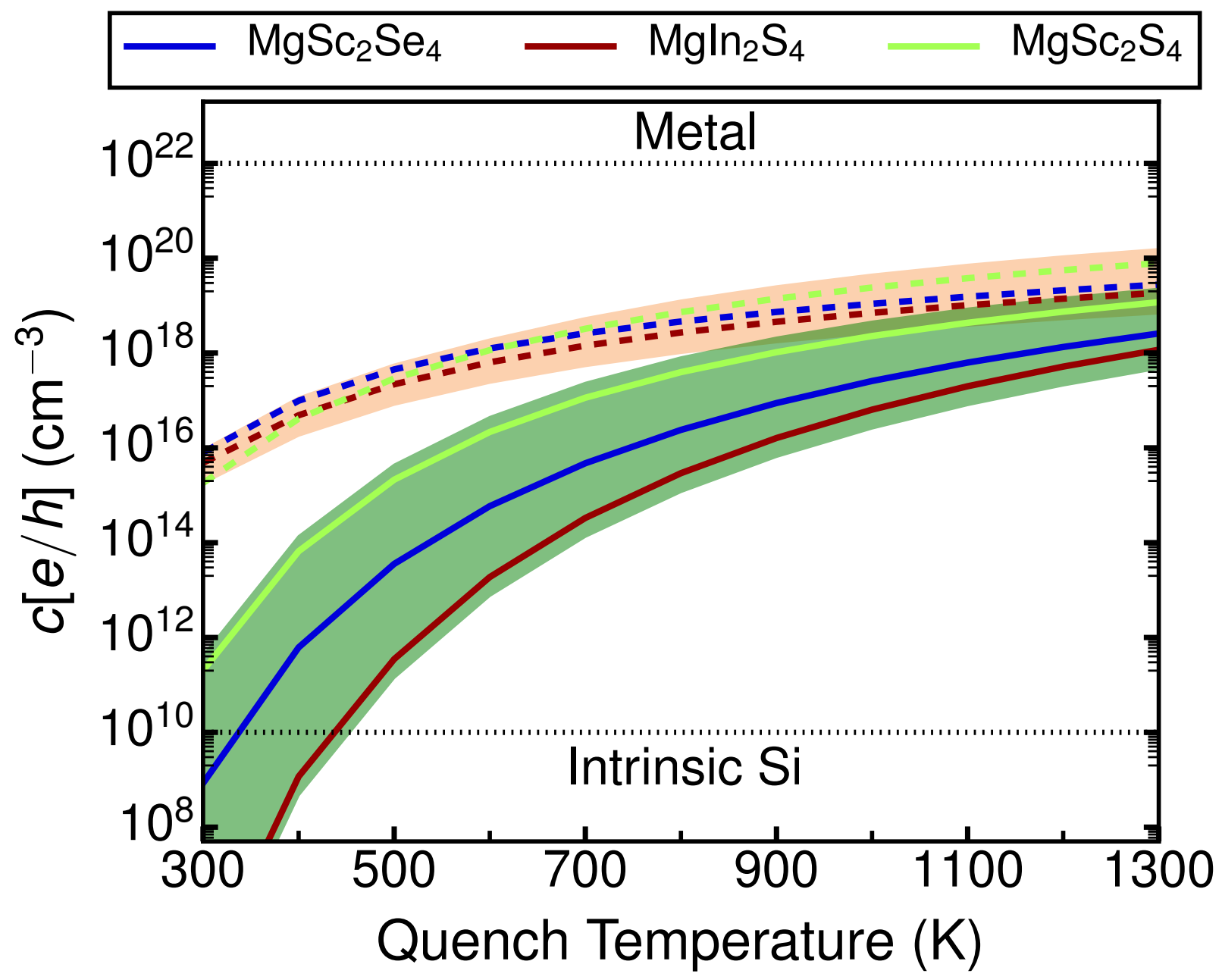

Figure 7: Free electron or hole concentrations $c[e / h]$ at $300 \mathrm{~K}$ as a function of temperature at which defect concentrations are quenched from. Solid and dashed lines indicate anionrich (green-shaded) and anion-poor (orange-shaded) regions, respectively. The blue, red, and green line colors correspond to $\mathrm{MgSc}_{2} \mathrm{Se}_{4}, \mathrm{MgIn}_{2} \mathrm{~S}_{4}$, and $\mathrm{MgSc}_{2} \mathrm{~S}_{4}$. The dotted black lines indicate the typical free carrier concentration in intrinsic $\mathrm{Si}\left(10^{10} \mathrm{~cm}^{-3}\right)$ and in metals $\left(10^{22} \mathrm{~cm}^{-3}\right)$. The $y$-axis values at $300 \mathrm{~K}$ are the $c[e / h]^{e q}$ for each spinel and values at $1300 \mathrm{~K}$ should indicate $c[e / h]^{\text {frozen }}$ corresponding to quenched defect concentrations from typical synthesis temperatures. ${ }^{22}$ For the case of anion-rich $\mathrm{MgIn}_{2} \mathrm{~S}_{4}$, the free carrier concentration is taken as the maximum of free electron and hole concentrations at each quench temperature.

than by Sc. 


\subsection{Chemical driving forces for anti-site defect formation}

In the previous sections and Table 1, we have demonstrated that the dominant defects in the chalcogenide spinels are anti-sites, signifying that $p$-type $\mathrm{Mg}_{\mathrm{In}}^{\prime}$ and $n$-type $\mathrm{In}_{\mathrm{Mg}}^{\bullet}$ are the primary intrinsic defects in $\mathrm{MgIn}_{2} \mathrm{~S}_{4}$, while $\mathrm{Sc}_{\mathrm{Mg}}^{\bullet}$ or $\mathrm{Mg}_{\mathrm{Sc}}^{\prime}$ are the main defects in $\mathrm{MgSc}_{2} \mathrm{~S}_{4}\left(\mathrm{Se}_{4}\right)$.

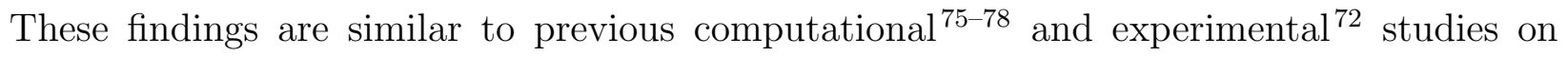
ternary oxide spinels, with anti-sites dominating over other intrinsic defects, i.e. vacancies and interstitials. Given that the $p$-type ( $n$-type) anti-site can compensate the excess electron (hole) ionized from the oppositely charged $n$-type ( $p$-type) anti-site, the resulting Fermi level and the concentration of free electrons (or holes) at equilibrium depends on the difference in concentration between the $p$ - and $n$-type anti-sites. For example, our data shows that under S-rich conditions an equal concentration of $p$ - and $n$-type defects (as indicated by $E_{F e r m i}^{e q}$ and $c[e / h]^{e q}$ in $\operatorname{MgIn}_{2} \mathrm{~S}_{4}$, Table 1 and Table S1), will pin the Fermi level within the band gap corresponding to a low concentration of free carriers.

So far, our calculations suggest that anti-site concentrations (and the corresponding difference between the concentration of $p$ - and $n$-type anti-sites) can be markedly different for the spinels considered in this work. Particularly, the results presented in Table 1 demonstrate that the concentration of anti-sites in $\mathrm{MgIn}_{2} \mathrm{~S}_{4}$ is always greater by several orders of magnitude (across all chemical conditions, see Table $\mathrm{S} 1$ ) than in $\mathrm{MgSc}_{2} \mathrm{~S}_{4}\left(\mathrm{Se}_{4}\right)$. Additionally, our calculations indicate that the difference between the $p$ - and $n$-type anti-site concentrations

in $\mathrm{MgIn}_{2} \mathrm{~S}_{4}$ is consistently lower than the Sc-spinels (Table S1), with profound effects on the type and magnitude of the electrical conductivity in the corresponding systems. Here, we rationalize the chemical factors driving such differences.

In general, the occurrence of anti-sites depends on a combination of several factors, such as, i) steric effects (i.e. the strain due to differences in ionic radii of the cations forming anti-sites), $i i$ ) the band-gap of each material (ease of ionizing the excess electron/hole), and iii) the electronic nature or bond character of specific bonds (covalent or ionic).

i Steric effects: anti-sites are facilitated if the cations substituting for each other possess 
similar ionic size. For example, $\mathrm{Mg}^{2+}$ has an ionic radius of $\sim 0.57 \AA$ and $\sim 0.72 \AA$ in tetrahedral and octahedral coordination, respectively, which compares well with the ionic radius of $\mathrm{In}^{3+} \sim 0.62 \AA$ in tetrahedral and $\sim 0.80 \AA$ in octahedral coordination, respectively, ${ }^{79}$ implying the facile formation of both $\mathrm{Mg}_{\mathrm{In}}^{\prime}$ and $\mathrm{In}_{\mathrm{Mg}}^{\bullet}$ anti-sites. While $\mathrm{Sc}^{3+}$ has an ionic radius of $\sim 0.75 \AA$ in octahedral sites, ${ }^{79}$ it has never been observed in tetrahedral coordination to our knowledge.

ii Band-gap: large band gaps in materials limit the possibility of ionization of the excess charge in defects, penalizing the injection of a free hole (electron) into the valence (conduction) band. Thus, the "large" band gap in $\mathrm{MgIn}_{2} \mathrm{~S}_{4}$ (Figure S3 in the SI) indicates a high energy penalty to ionize the excess charge, forcing $\mathrm{Mg}_{\mathrm{In}}^{\prime}$ to charge compensate $\mathrm{In}_{\mathrm{Mg}}^{\bullet}$ (and vice-versa) and leading to a lower difference in concentration between $\mathrm{Mg}_{\mathrm{In}}^{\prime}$ and $\mathrm{In}_{\mathrm{Mg}}^{\bullet}$ across all conditions (Table S1). In contrast, the band gaps in Sc-spinels are quantitatively lower than the In-spinel, indicating that the energy penalty for either $\mathrm{Sc}_{\mathrm{Mg}}^{\bullet}$ or $\mathrm{Mg}_{\mathrm{Sc}}^{\prime}$ to ionize the excess charge is significantly smaller, suggesting that the $\mathrm{Mg}_{\mathrm{Sc}}^{\prime}$ may not be required to charge compensate the $\mathrm{Sc}_{\mathrm{Mg}}^{\bullet}$. Since inversion in the spinel structure correlates with the formation of comparable quantities of both $p$ - and $n$-type anti-sites, $\mathrm{MgIn}_{2} \mathrm{~S}_{4}$ is more susceptible in exhibiting spinel inversion than the Sc-compounds, in agreement with previous experimental studies. ${ }^{65}$

iii Bond character: covalent bonds with significant hybridization of the transition metal and the anion can tolerate anti-sites better than ionic bonds, due to greater electrostatic screening of the excess charge in the anti-sites. Since each octahedral $(16 d)$ site in the spinel structure shares edges with 6 other $16 d$ sites (Figure 2), ${ }^{80}$ electrostatic screening will be important in stabilizing the $p$-type anti-sites (i.e., $\mathrm{Mg}_{\mathrm{In}}^{\prime}$ and $\mathrm{Mg}_{\mathrm{Sc}}^{\prime}$ ). From a qualitative analysis of the valence band edge in the density of states (Figure S3 in the $\mathrm{SI})$ in $\mathrm{MgSc}_{2} \mathrm{~S}_{4}\left(\mathrm{Se}_{4}\right)$, we speculate that both $\mathrm{Mg}$ and $\mathrm{Sc}$ bond quite ionically with the anion (S/Se). In contrast, the In-S bonds show significant hybridization in $\mathrm{MgIn}_{2} \mathrm{~S}_{4}$ 
compared to the Sc-S(Se) bonds in $\mathrm{MgSc}_{2} \mathrm{~S}_{4}\left(\mathrm{Se}_{4}\right)$, stabilizing the $p$-type $\mathrm{Mg}_{\text {In }}^{\prime}$. These observations could explain the higher concentrations of $\mathrm{Mg}_{\mathrm{In}}^{\prime}$ as opposed to $\mathrm{Mg}_{\mathrm{Sc}}^{\prime}$, as indicated by our calculations across all conditions (Table S1).

From this analysis, two criteria to design a ternary spinel ionic conductor with minimal electronic conductivity emerge: $i$ ) materials with large band-gap (curbing the ionization of free carriers) and $i$ i) materials where both $p$ - and $n$-type anti-sites are equally likely to form (leading to spinel inversion and lower free carriers) are preferable.

\subsection{Extrinsic doping}

Aliovalent doping of ionic conductors can be used as a strategy to both enhance the ionic conductivities while suppressing intrinsic electronic (hole) conductivities. We explored the defect chemistry of extrinsic dopants in the $\gamma$ facet of $\mathrm{MgSc}_{2} \mathrm{Se}_{4}$ (Figure 6), comprising tetravalent and pentavalent non-redox cation substitution on Sc as well as halogen doping on Se. Given that the intrinsic $E_{F e r m i}^{e q}$ is $\sim 1.08 \mathrm{eV}$ in anion-poor $\mathrm{MgSc}_{2} \mathrm{Se}_{4}$, doping of most cations is not energetically favored (Figure 6a), with the exception of Ce, Ti, and Zr. Halogen doping on Se appears feasible $\left(E_{f}<0.4 \mathrm{eV}\right.$, Figure $\left.6 \mathrm{~b}\right)$, although it may further increase the $n$-type behavior. In contrast, $n$-type Ce and Ti have their respective donor transition levels deeper in the band-gap (Figure 6a) and may reduce the free electron concentration in $\mathrm{MgSc}_{2} \mathrm{Se}_{4}$. However, it remains to be experimentally confirmed whether $\mathrm{Ti}$ and $\mathrm{Ce}$ can be efficiently doped on the Sc site.

\section{Conclusion}

Using first-principles calculations, we have analyzed the role of defect chemistry in influencing the electrical conductivities of three chalcogenide spinels, $\mathrm{MgSc}_{2} \mathrm{Se}_{4}, \mathrm{MgSc}_{2} \mathrm{~S}_{4}$ and $\mathrm{MgIn}_{2} \mathrm{~S}_{4}$, which are potential Mg-ion conductors. We find that intrinsic point defects, such as Mg-metal anti-sites $\left(\{\mathrm{Sc} / \mathrm{In}\}_{\mathrm{Mg}}^{\bullet}, \mathrm{Mg}_{\{\mathrm{Sc} / \mathrm{In}\}}^{\prime}\right)$ and Mg-vacancies $\left(\mathrm{Vac}_{\mathrm{Mg}}^{\prime \prime}\right)$, dramatically affect 
the free carrier concentrations of the spinels under consideration. Additionally, controlling the anion-content during synthesis is an important factor in determining the defect energetics and the resultant electrical conductivity, with all three spinels exhibiting high $n$-type conductivity in anion-poor conditions and marginal $p$-type behavior in anion-rich conditions. Also, fast cooling leads to large concentrations of intrinsic defects being quenched within the structure, which can increase both the free hole (anion-rich) and free electron (anion-poor) concentrations in $\mathrm{MgSc}_{2} \mathrm{Se}_{4}, \mathrm{MgSc}_{2} \mathrm{~S}_{4}$ and $\mathrm{MgIn}_{2} \mathrm{~S}_{4}$. Hence, the lowest electronic conductivity is to be expected for samples synthesized under anion-excess, and slowly cooled to room temperature. Among the three structures considered, $\mathrm{MgIn}_{2} \mathrm{~S}_{4}$ exhibits the lowest free carrier concentration across various conditions, largely due to inversion within the spinel. Finally, the introduction of aliovalent dopants, such as Ce and Ti on Sc, may mitigate the electronic conductivity observed in $\mathrm{MgSc}_{2} \mathrm{Se}_{4}$. Our work indicates the importance of defects in the field of solid electrolytes, and the framework used here can be applied to other systems as well, which will eventually aid both in the calibration of existing candidates and accelerated materials discovery.

\section{Acknowledgement}

This work was fully supported as part of the Joint Center for Energy Storage Research (JCESR), an Energy Innovation Hub funded by the U.S. Department of Energy, Office of Science, and Basic Energy Sciences. This study was supported by Subcontract 3F-31144. The authors thank the National Energy Research Scientific Computing Center (NERSC) for providing computing resources. The authors thank Mark D. Asta at UC Berkeley for constructive comments, while PC acknowledges Jacques-Arnaud Dâwson for useful discussion. 


\section{References}

(1) Hsu, K. F. Cubic AgPb $\mathrm{SbTe}_{2+m}$ : Bulk Thermoelectric Materials with High Figure of Merit. Science 2004, 303, 818-821.

(2) Snyder, G. J.; Toberer, E. S. Complex thermoelectric materials. Nat Mater 2008, 7, $105-114$.

(3) Persson, C.; Zhao, Y.-J.; Lany, S.; Zunger, A. n-type doping of CuInSe 2 and $\mathrm{CuGaSe}_{2}$. Phys. Rev. B 2005, 72.

(4) Todorov, T. K.; Reuter, K. B.; Mitzi, D. B. High-Efficiency Solar Cell with EarthAbundant Liquid-Processed Absorber. Adv Mater 2010, 22, E156-E159.

(5) Chen, S.; Walsh, A.; Luo, Y.; Yang, J.-H.; Gong, X. G.; Wei, S.-H. Wurtzite-derived polytypes of kesterite and stannite quaternary chalcogenide semiconductors. Phys Rev $B$ 2010, 82.

(6) Sun, R.; Chan, M. K. Y.; Kang, S.; Ceder, G. Intrinsic stoichiometry and oxygeninduced $p$-type conductivity of pyrite $\mathrm{FeS}_{2}$. Phys Rev B 2011, 84 .

(7) Sun, R.; Ceder, G. Feasibility of band gap engineering of pyrite $\mathrm{FeS}_{2}$. Phys Rev B 2011, 84.

(8) Redinger, A.; Berg, D. M.; Dale, P. J.; Siebentritt, S. The Consequences of Kesterite Equilibria for Efficient Solar Cells. J Am Chem Soc 2011, 133, 3320-3323.

(9) Baranowski, L. L.; Zawadzki, P.; Christensen, S.; Nordlund, D.; Lany, S.; Tamboli, A. C.; Gedvilas, L.; Ginley, D. S.; Tumas, W.; Toberer, E. S.; Zakutayev, A. Control of Doping in $\mathrm{Cu}_{2} \mathrm{SnS}_{3}$ through Defects and Alloying. Chem Mater 2014, 26, 4951-4959.

(10) Kitchaev, D. A.; Ceder, G. Evaluating structure selection in the hydrothermal growth of $\mathrm{FeS}_{2}$ pyrite and marcasite. Nat. Comms. 2016, 7, 13799. 
(11) Butler, K. T.; McKechnie, S.; Azarhoosh, P.; van Schilfgaarde, M.; Scanlon, D. O.; Walsh, A. Quasi-particle electronic band structure and alignment of the V-VI-VII semiconductors SbSI, SbSBr, and SbSeI for solar cells. Appl. Phys. Lett. 2016, 108, 112103.

(12) Patrie, M.; Flahaut, J.; Domage, L. Chimie Minerale - Sur Une Nouvelle Serie De Spinelles Soufres Contenant Des Terres Rares Ou Du Scandium. C. R. Hebd. Acad. Sci. 1964, 258, 2585-2586.

(13) Guittard, M.; Souleau, C.; Farsam, H. Sur Une Nouvelle Serie De Spinelles Selenies Des Terres Rares De l'yttrium Et Du Scandium. C. R. Hebd. Acad. Sci. 1964, 259, $2847-2849$.

(14) Gamble, F. R.; DiSalvo, F. J.; Klemm, R. A.; Geballe, T. H. Superconductivity in Layered Structure Organometallic Crystals. Science 1970, 168, 568-570.

(15) Mattheiss, L. F. Band Structures of Transition-Metal-Dichalcogenide Layer Compounds. Phys Rev B 1973, 8, 3719-3740.

(16) Williams, A. J.; McQueen, T. M.; Cava, R. J. The stoichiometry of FeSe. Solid State Commun 2009, 149, 1507-1509.

(17) Whittingham, M. S. Chemistry of intercalation compounds: Metal guests in chalcogenide hosts. Prog. Solid State Ch. 1978, 12, 41-99.

(18) Levi, E.; Gershinsky, G.; Aurbach, D.; Isnard, O.; Ceder, G. New Insight on the Unusually High Ionic Mobility in Chevrel Phases. Chem Mater 2009, 21, 1390-1399.

(19) Kamaya, N.; Homma, K.; Yamakawa, Y.; Hirayama, M.; Kanno, R.; Yonemura, M.; Kamiyama, T.; Kato, Y.; Hama, S.; Kawamoto, K.; Mitsui, A. A lithium superionic conductor. Nat Mater 2011, 10, 682-686.

(20) Ong, S. P.; Mo, Y.; Richards, W. D.; Miara, L.; Lee, H. S.; Ceder, G. Phase stability, electrochemical stability and ionic conductivity of the $\mathrm{Li}_{10 \pm 1} \mathrm{MP}_{2} \mathrm{X}_{12}(\mathrm{M}=\mathrm{Ge}, \mathrm{Si}, \mathrm{Sn}$, 
$\mathrm{Al}$ or $\mathrm{P}$, and $\mathrm{X}=\mathrm{O}, \mathrm{S}$ or $\mathrm{Se}$ ) family of superionic conductors. Energy Environ Sci 2013, 6, 148-156.

(21) Wang, Y.; Richards, W. D.; Ong, S. P.; Miara, L. J.; Kim, J. C.; Mo, Y.; Ceder, G. Design principles for solid-state lithium superionic conductors. Nat Mater 2015, 14 , $1026-1031$.

(22) Canepa, P.; Bo, S.-H.; Gopalakrishnan, S. G.; Key, B.; Richards, W. D.; Tan, S.; Tian, Y.; Wang, Y.; Li, J.; Ceder, G. High Magnesium Mobility in Solids: An Investigation of Ternary Spinel Chalcogenides. submitted 2017,

(23) Nozik, A. Quantum dot solar cells. Physica E 2002, 14, 115-120.

(24) Eisler, H.-J.; Sundar, V. C.; Bawendi, M. G.; Walsh, M.; Smith, H. I.; Klimov, V. Color-selective semiconductor nanocrystal laser. Appl Phys Lett 2002, 80, 4614.

(25) Talapin, D. V.; Murray, C. B. PbSe Nanocrystal Solids for $n$ - and $p$-Channel Thin Film Field-Effect Transistors. Science 2005, 310, 86-89.

(26) Anikeeva, P. O.; Halpert, J. E.; Bawendi, M. G.; Bulović, V. Quantum Dot LightEmitting Devices with Electroluminescence Tunable over the Entire Visible Spectrum. Nano Lett 2009, 9, 2532-2536.

(27) Meinardi, F.; Colombo, A.; Velizhanin, K. A.; Simonutti, R.; Lorenzon, M.; Beverina, L.; Viswanatha, R.; Klimov, V. I.; Brovelli, S. Large-area luminescent solar concentrators based on 'Stokes-shift-engineered' nanocrystals in a mass-polymerized PMMA matrix. Nature Photon. 2014, 8, 392-399.

(28) Jia, S.; Ji, H.; Climent-Pascual, E.; Fuccillo, M. K.; Charles, M. E.; Xiong, J.; Ong, N. P.; Cava, R. J. Low-carrier-concentration crystals of the topological insulator $\mathrm{Bi}_{2} \mathrm{Te}_{2}$ Se. Phys Rev B 2011, 84 . 
(29) Chhowalla, M.; Shin, H. S.; Eda, G.; Li, L.-J.; Loh, K. P.; Zhang, H. The chemistry of two-dimensional layered transition metal dichalcogenide nanosheets. Nat Chem 2013, 5, 263-275.

(30) Behrh, G. K.; Isobe, M.; Massuyeau, F.; Serier-Brault, H.; Gordon, E. E.; Koo, H.J.; Whangbo, M.-H.; Gautier, R.; Jobic, S. Oxygen-Vacancy-Induced Midgap States Responsible for the Fluorescence and the Long-Lasting Phosphorescence of the Inverse Spinel $\mathrm{Mg}(\mathrm{Mg}, \mathrm{Sn}) \mathrm{O}_{4}$. Chem Mater 2017, 29, 1069-1075.

(31) Griffin, S. M.; Reidulff, M.; Selbach, S. M.; Spaldin, N. A. Defect chemistry as a crystal structure design parameter: Intrinsic Point defects and Ga substitution in $\mathrm{InMnO}_{3}$. Chem Mater 2017,

(32) Canepa, P.; Gautam, G. S.; Hannah, D. C.; Malik, R.; Liu, M.; Gallagher, K. G.; Persson, K. A.; Ceder, G. Odyssey of Multivalent Cathode Materials: Open Questions and Future Challenges. Chem Rev 2017, 117, 4287-4341.

(33) Freysoldt, C.; Grabowski, B.; Hickel, T.; Neugebauer, J.; Kresse, G.; Janotti, A.; de Walle, C. G. V. First-principles calculations for point defects in solids. Rev Mod Phys 2014, 86, 253-305.

(34) Zhang, S.; Northrup, J. Chemical potential dependence of defect formation energies in GaAs: Application to Ga self-diffusion. Phys. Rev. Lett. 1991, 67, 2339-2342.

(35) Laks, D. B.; de Walle, C. G. V.; Neumark, G. F.; Blöchl, P. E.; Pantelides, S. T. Native defects and self-compensation in ZnSe. Phys. Rev. B 1992, 45, 10965-10978.

(36) Kwak, K. W.; Vanderbilt, D.; King-Smith, R. D. First-principles study of phosphorus and nitrogen impurities in ZnSe. Phys. Rev. B 1995, 52, 11912-11919.

(37) de Walle, C. G. V.; Neugebauer, J. First-principles calculations for defects and impurities: Applications to III-nitrides. J. Appl. Phys. 2004, 95, 3851-3879. 
(38) Freysoldt, C.; Neugebauer, J.; de Walle, C. G. V. Fully Ab Initio Finite-Size Corrections for Charged-Defect Supercell Calculations. Phys Rev Lett 2009, 102.

(39) Freysoldt, C.; Neugebauer, J.; de Walle, C. G. V. Electrostatic interactions between charged defects in supercells. Physica Status Solidi B 2010, 248, 1067-1076.

(40) Broberg, D.; Medasani, B.; Zimmermann, N.; Canning, A.; Haranczyk, M.; Asta, M.; Hautier, G. PyCDT: A Python toolkit for modeling point defects in semiconductors and insulators. under review in Comput. Phys. Commun. 2016,

(41) Komsa, H.-P.; Rantala, T. T.; Pasquarello, A. Finite-size supercell correction schemes for charged defect calculations. Phys Rev B 2012, 86 .

(42) Leslie, M.; Gillan, N. J. The energy and elastic dipole tensor of defects in ionic crystals calculated by the supercell method. J Phys C: Solid State Phys 1985, 18, 973-982.

(43) Makov, G.; Payne, M. C. Periodic boundary conditions inab initiocalculations. Phys Rev B 1995, 51, 4014-4022.

(44) Carloni, P.; Blöechl, P. E.; Parrinello, M. Electronic structure of the $\mathrm{Cu}$, Zn superoxide dismutase active site and its interactions with the substrate. J Phys Chem 1995, 99, $1338-1348$.

(45) Schultz, P. A. Charged Local Defects in Extended Systems. Phys Rev Lett 2000, 84, $1942-1945$.

(46) Lany, S.; Zunger, A. Assessment of correction methods for the band-gap problem and for finite-size effects in supercell defect calculations: Case studies for $\mathrm{ZnO}$ and GaAs. Phys. Rev. B 2008, 78.

(47) Hohenberg, P.; Kohn, W. Inhomogeneous Electron Gas. Phys Rev 1964, 136, B864B871. 
(48) Kohn, W.; Sham, L. J. Self-Consistent Equations Including Exchange and Correlation Effects. Phys Rev 1965, 140, A1133-A1138.

(49) Perdew, J. P.; Burke, K.; Ernzerhof, M. Generalized Gradient Approximation Made Simple. Phys Rev Lett 1996, 77, 3865-3868.

(50) Kresse, G.; Hafner, J. Ab initio molecular dynamics for liquid metals. Phys Rev B 1993, 47, 558-561.

(51) Kresse, G.; Furthmüller, J. Efficient iterative schemes forab initiototal-energy calculations using a plane-wave basis set. Phys Rev B 1996, 54, 11169-11186.

(52) Blöchl, P. E. Projector augmented-wave method. Phys Rev B 1994, 50, 17953-17979.

(53) Kresse, G.; Joubert, D. From ultrasoft pseudopotentials to the projector augmentedwave method. Phys Rev B 1999, 59, 1758-1775.

(54) Monkhorst, H. J.; Pack, J. D. Special points for Brillouin-zone integrations. Phys Rev $B$ 1976, 13, 5188-5192.

(55) Ong, S. P.; Richards, W. D.; Jain, A.; Hautier, G.; Kocher, M.; Cholia, S.; Gunter, D.; Chevrier, V. L.; Persson, K. A.; Ceder, G. Python Materials Genomics (pymatgen): A robust, open-source python library for materials analysis. Computational Materials Science 2013, 68, 314-319.

(56) Jain, A.; Ong, S. P.; Hautier, G.; Chen, W.; Richards, W. D.; Dacek, S.; Cholia, S.; Gunter, D.; Skinner, D.; Ceder, G.; Persson, K. A. Commentary: The Materials Project: A materials genome approach to accelerating materials innovation. APL Materials 2013, 1, 011002.

(57) Heyd, J.; Scuseria, G. E.; Ernzerhof, M. Hybrid functionals based on a screened Coulomb potential. The Journal of Chemical Physics 2003, 118, 8207-8215. 
(58) Heyd, J.; Scuseria, G. E.; Ernzerhof, M. Erratum: "Hybrid functionals based on a screened Coulomb potential" [J. Chem. Phys. 118, 8207 (2003)]. The Journal of Chemical Physics 2006, 124, 219906.

(59) Lejaeghere, K. et al. Reproducibility in density functional theory calculations of solids. Science 2016, 351, aad3000-aad3000.

(60) Wang, C. S.; Pickett, W. E. Density-Functional Theory of Excitation Spectra of Semiconductors: Application to Si. Phys. Rev. Lett. 1983, 51, 597-600.

(61) Chevrier, V. L.; Ong, S. P.; Armiento, R.; Chan, M. K. Y.; Ceder, G. Hybrid density functional calculations of redox potentials and formation energies of transition metal compounds. Phys Rev B 2010, 82.

(62) Gastaldi, L.; Lapiccirella, A. Three different methods of determining the cation distribution in spinels: A comparison. J Solid State Chem 1979, 30, 223-229.

(63) Weil, J. A. A review of electron spin spectroscopy and its application to the study of paramagnetic defects in crystalline quartz. Physics and Chemistry of Minerals 1984, $10,149-165$.

(64) Livraghi, S.; Paganini, M. C.; Giamello, E.; Selloni, A.; Valentin, C. D.; Pacchioni, G. Origin of Photoactivity of Nitrogen-Doped Titanium Dioxide under Visible Light. J Am Chem Soc 2006, 128, 15666-15671.

(65) Wakaki, M.; Shintani, O.; Ogawa, T.; Arai, T. Optical and Electrical Properties of Inverse Spinel Compound $\mathrm{MgIn}_{2} \mathrm{~S}_{4}$. Japanese Journal of Applied Physics 1980, 19, 255.

(66) Miara, L. J.; Richards, W. D.; Wang, Y. E.; Ceder, G. First-Principles Studies on Cation Dopants and Electrolyte Cathode Interphases for Lithium Garnets. Chem Mater 2015, 27, 4040-4047. 
(67) Janek, J.; Zeier, W. G. A solid future for battery development. Nature Energy 2016, $1,16141$.

(68) Kato, Y.; Hori, S.; Saito, T.; Suzuki, K.; Hirayama, M.; Mitsui, A.; Yonemura, M.; Iba, H.; Kanno, R. High-power all-solid-state batteries using sulfide superionic conductors. Nature Energy 2016, 1, 16030.

(69) Pauling, L. The theoretical prediction of the physical properties of many-electron atoms and ions. Mole refraction, diamagnetic susceptibility, and extension in space. Proceedings of the Royal Society of London. Series A, Containing Papers of a Mathematical and Physical Character 1927, 114, 181-211.

(70) Burdett, J. K.; Price, G. D.; Price, S. L. Factors influencing solid-state structure - an analysis using pseudopotential radii structural maps. Phys Rev B 1981, 24, 2903-2912.

(71) Burdett, J. K.; Price, G. D.; Price, S. L. Role of the crystal-field theory in determining the structures of spinels. J Am Chem Soc 1982, 104, 92-95.

(72) Sickafus, K. E.; Wills, J. M.; Grimes, N. W. Structure of Spinel. J Am Ceram Soc 2004, 82, 3279-3292.

(73) Zhang, X.; Zunger, A. Diagrammatic Separation of Different Crystal Structures of $\mathrm{A}_{2} \mathrm{BX}_{4}$ Compounds Without Energy Minimization: A Pseudopotential Orbital Radii Approach. Adv. Funct. Mater. 2010, 20, 1944-1952.

(74) Lucero, M. J.; Aguilera, I.; Diaconu, C. V.; Palacios, P.; Wahnón, P.; Scuseria, G. E. Screened hybrid and self-consistentGWcalculations of cadmium/magnesium indium sulfide materials. Phys Rev B 2011, 83.

(75) Stevanovi ć, V.; dAvezac, M.; Zunger, A. Simple Point-Ion Electrostatic Model Explains the Cation Distribution in Spinel Oxides. Phys. Rev. Lett. 2010, 105, 075501. 
(76) Paudel, T. R.; Zakutayev, A.; Lany, S.; d'Avezac, M.; Zunger, A. Doping Rules and Doping Prototypes in $\mathrm{A}_{2} \mathrm{BO}_{4}$ Spinel Oxides. Adv. Funct. Mater. 2011, 21, 4493-4501.

(77) Stevanović, V.; dAvezac, M.; Zunger, A. Universal Electrostatic Origin of Cation Ordering in $\mathrm{A}_{2} \mathrm{BO}_{4}$ Spinel Oxides. J. Am. Chem. Soc. 2011, 133, 11649-11654.

(78) Das, D.; Ghosh, S. First-principles investigations into the thermodynamics of cation disorder and its impact on electronic structure and magnetic properties of spinel Co $\left(\mathrm{Cr}_{1-x} \mathrm{Mn}_{x}\right)_{2} \mathrm{O}_{4}$. J. Phys.: Condens. Matter 2016, 29, 055805.

(79) Shannon, R. D. Revised effective ionic radii and systematic studies of interatomic distances in halides and chalcogenides. Acta Cryst. Sect. A 1976, 32, 751-767.

(80) Gautam, G. S.; Canepa, P.; Urban, A.; Bo, S.-H.; Ceder, G. Influence of Inversion on Mg Mobility and Electrochemistry in Spinels. Chemistry of Materials 2017, 29, 7918-7930. 